\title{
Component sizing of a plug-in hybrid electric powertrain via convex optimization
}

\author{
Nikolce Murgovski $^{1, \mathrm{a}}$, Lars Johannesson ${ }^{\mathrm{a}, \mathrm{b}}$, Jonas Sjöberg ${ }^{\mathrm{a}}$, Bo Egardt ${ }^{\mathrm{a}}$ \\ ${ }^{a}$ Department of Signals and Systems, Charmers University of Technology, 41296 Gothenburg, Sweden \\ ${ }^{b}$ Viktoria Institute, 41756 Gothenburg, Sweden
}

\begin{abstract}
This paper presents a novel convex modeling approach which allows for a simultaneous optimization of battery size and energy management of a plug-in hybrid powertrain by solving a semidefinite convex problem. The studied powertrain belongs to a city bus which is driven along a perfectly known bus line with fixed charging infrastructure. The purpose of the paper is to present the convexifying methodology and validate the necessary approximations by comparing with results obtained by Dynamic programming when using the original nonlinear, non-convex, mixed-integer models. The comparison clearly shows the importance of the gear and engine on/off decisions, and it also shows that the convex optimization and Dynamic Programming point toward similar battery size and operating cost when the same gear and engine on/off heuristics are used. The main conclusion in the paper is that due to the low computation time, the convex modeling approach enables optimization of problems with two or more state variables, e.g. allowing for thermal models of the components; or to include more sizing variables, e.g. sizing of the engine and the electric machine simultaneously.
\end{abstract}

Keywords:

plug-in hybrid electric vehicle, slide-in electric vehicle, battery sizing, power management, convex optimization

\section{Introduction}

Hybrid Electric Vehicles (HEVs) are widely regarded as one of the most promising means for achieving a near-term reduction of emissions and energy consumption from the transportation sector. HEV powertrains include an internal combustion engine (ICE), one or more electric machines (EMs), and an energy buffer, typically a battery and/or a super capacitor, which depending on their configuration are commonly divided in three different topologies: series, parallel and series-parallel. The powertrain topologies mainly differ in the available degree of freedom in choosing the ICE operating point, but their capability to improve energy consumption can be generally described by 1) the possibility to recover braking energy by using the EMs as generators and storing the energy in the buffer, 2) ability to shut down the ICE during idling and low load demands and 3) the possibility to run the ICE at more efficient load conditions while storing the excess energy in the buffer. For a detailed overview on hybrid vehicles, see e.g. [1].

The so-called plug-in HEVs (PHEVs) are equipped with a charging connector (typically an on-board charger), which allows the PHEVs to charge the electric buffer from the grid. PHEVs are designed to be charged either by a standard household electric power infrastructure or at stations installed on, e.g. parking lots, shopping malls, or other locations.

Email addresses: nikolce.murgovski@chalmers.se (Nikolce Murgovski), larsjo@chalmers.se, lars.johannesson@viktoria.se (Lars Johannesson), jonas.sjoberg@chalmers.se (Jonas Sjöberg), bo.egardt@chalmers.se (Bo Egardt)

${ }^{1}$ Corresponding author, Tel: +46 317724800 , Fax: +46 317721748
In recent years, PHEVs have been considered for use in public transportation by equipping high traffic bus lines with a charging infrastructure [2, 3], offering a flexible crossbreed between an HEV city bus and a trolley-bus. In [2] the PHEV city bus is, while driving, inductively charged from underground cables that have been buried along sections of the bus line. In [3] the PHEV is equipped with a super capacitor which is charged at bus stops through a docking station. Since the PHEVs are to be charged at relatively high power, the energy buffer makes it possible to drive a significant part of the bus line on electric power even though the charging infrastructure might be sparsely distributed.

The cost optimal sizing of the energy buffer, i.e. determining power rating and energy capacity of these PHEVs, is heavily dependent on the charging infrastructure, the drive pattern and the topography along the bus line. The solution to this optimization problem, however, depends not only on the city bus system configuration and the cost of the on-board electric components, but also on changing factors such as fuel and electricity prices. Moreover, a complicating issue when evaluating HEV city buses is that the energy efficiency of the powertrain depends on how well adapted the energy management strategy is to the bus line [4]. For PHEV city buses the energy management strategy decides the operating point of the ICE and thereby when and at which rate the energy buffer is to be discharged. When optimizing the PHEV public transportation system based on a dynamic model of the powertrain, a badly tuned energy management may lead to a non-optimal sizing of the energy buffer [5].

There are two main approaches to the problem of optimal siz- 


\begin{tabular}{|c|c|c|c|}
\hline & $v$ & Velocity. \\
\hline$A_{f}$ & Nomenclature & $w$ & Penalty coefficient. \\
\hline E & Specific energy. & $x_{c s}$ & PHEV charging rate. \\
\hline$F$ & Force. & $y_{b}$ & Battery calendar life. \\
\hline$I$ & Rotational inertia. & $y_{p}$ & Battery payback period. \\
\hline$J$ & Cost function. & & Time interval. \\
\hline$M$ & Efficiency matrix for feasible gears. & $\alpha$ & Slope of the road. \\
\hline$N$ & Number of time samples. & $\beta_{1}, \beta_{2}$ & Parameters shaping the EGU efficiency. \\
\hline$P$ & Power. & $\gamma$ & Gear. \\
\hline$Q$ & Battery cell capacity. & $\eta$ & Efficiency. \\
\hline$Q_{f}$ & Energy content of the fuel. & $\rho_{\text {air }}$ & Air density. \\
\hline & Ah-throughput of the cell. & $\omega$ & Angular velocity. \\
\hline$R$ & Battery cell resistance. & \multicolumn{2}{|c|}{ Subscripts } \\
\hline $\mathbb{R}$ & Set of real numbers. & 0 & Initial value. \\
\hline $\begin{array}{l}R_{w} \\
T\end{array}$ & Wheel radius. & $D P$ & Dynamic Programming. \\
\hline $\begin{array}{l}1 \\
V_{o c}\end{array}$ & $\begin{array}{l}\text { Torque. } \\
\text { Open circuit voltage of the battery cell. }\end{array}$ & $E G U$ & Engine-generator Unit. \\
\hline$a_{0}, a_{1}$ & Parameters describing the EGU efficiency. & $E M$ & Electric Machine. \\
\hline$b_{0}, b_{1}, b_{2}$ & Parameters describing the EM efficiency. & $I C E$ & Internal Combustion Engine. \\
\hline$c_{0}, c_{1}, c_{2}$ & Parameters describing the ICE efficiency. & aux & Auxiliaries. \\
\hline$c_{b}, c_{b e}$ & Battery cost. & $b$ & Battery. \\
\hline$c_{d}$ & Drag coefficient. & base & Baseline powertrain. \\
\hline$c_{e l}$ & Electricity price. & $b c$ & Battery cell. \\
\hline$c_{f}$ & Fuel price. & brk & Brakes. \\
\hline & Rolling resistance coefficient. & $c s$ & Charging station. \\
\hline $\operatorname{dom} f$ & Domain of $f$. & dem & Demanded. \\
\hline$e$ & Engine state. & $d g$ & Differential gear. \\
\hline$g$ & Gravitational acceleration. & $f$ & Fuel. \\
\hline$h$ & Sampling interval. & $f l$ & Final value. \\
\hline$i$ & Current in the battery cell. & fric & Friction. \\
\hline$m$ & Mass. & grid & Electric grid. \\
\hline$n$ & Quantity. & idle & Engine idling. \\
\hline$p$ & Yearly interest rate for the battery payment. & loss & Power loss. \\
\hline$r$ & Gear ratio. & on & Engine on/off. \\
\hline$s$ & Average distanced traveled in one year. & $s$ & Crankshaft. \\
\hline$s o c$ & Battery state of charge. & up & Engine start-up (cranking). \\
\hline$t$ & Time. & veh & Vehicle. \\
\hline & Control input. & $w$ & Wheels. \\
\hline
\end{tabular}

ing and control of HEVs. The first approach relies on heuristic algorithms [6-13], while the second approach uses optimal control methods which give opportunity to evaluate various configurations on the basis of their optimal performance when simulated along one or several drive cycles (e.g. speed vs. time profiles).

From the optimal control methods, Dynamic Programming (DP) [14] is the most commonly used [15-20]. The main advantage with DP is the capability to use nonlinear, non-convex models of the components consisting of continuous and integer (mixed integer) optimization variables. Another important advantage is that the computation time increases linearly with the drive cycle length. However, DP has two important limitations when sizing powertrain components. The most serious limitation is that the computation time increases exponentially [14] with the number of state variables. As a consequence, the powertrain model is typically limited to only one or possibly two continuous state variables [6-13]. More than three state variables would be highly impractical requiring a dedicated optimization code and a computer cluster. Moreover, since DP operates by recursively solving a smaller subproblem for each time step, the second limitation of DP is that it is not possible to directly include the component sizing into the optimization. Instead, DP must be run in several loops to obtain the optimal control over a grid of component sizes.

The underlying research question in this paper is to investigate which approximations are needed in order to formulate the powertrain sizing and the corresponding optimal control problem as a convex optimization problem. The interest in convex modeling and optimization as a method for powertrain sizing is to avoid the limitations of DP and thereby to allow simultaneous optimization of parameters deciding the component sizes 
(e.g. engine, battery, electric machine, ...) for models with several state variables. This paper focuses only on the battery sizing problem although the long term research goal is to include the sizing of more powertrain components into the convex optimization problem.

Studying a PHEV city bus based on either a series or a parallel topology, this paper describes the modeling steps needed to formulate the energy management and battery sizing problem as a semidefinite convex problem [21]. This convex problem can then be efficiently solved for a global optimum using generally available solvers, SeDuMi [22], SDPT3 [23].

In the optimization approach the power characteristics of the engine, the engine-generator unit and the electric machines are approximated by a convex second order polynomial, and the convex battery model assumes quadratic losses. The only heuristic choice in the optimization is the gear selection and the engine on/off operation which are tuned in an outer optimization loop. The heuristics serves two purposes: it removes the need for solving a mixed integer problem; and at the same time it allows for a flexible approach to model the performance of the gearshift strategy which makes it possible to incorporate both physical constraints and limitations imposed by drivability considerations.

Two examples illustrate the methodology for the optimal PHEV battery design for a bus line with fixed charging infrastructure. The first example investigates if the convex problem results in a similar battery design compared to the non-convex mixed integer problem solved by DP. The results indicate that both optimization problems point toward a similar solution. The second example studies a PHEV city bus equipped with a dual battery comprised of both energy optimized cells and power optimized cells; a problem that can be solved in minutes with the convex optimization approach, but would require a significant computational effort with the DP approach. Besides demonstrating the utility of the convex modeling approach the example clearly shows the sensitivity of battery sizing with respect to battery prices and charging power.

The paper is outlined as follows: background on convex optimization is given in Section 2, the studied bus line and charging infrastructure are discussed in Section 3; the PHEV powertrain model is described in Section 4; problem formulation and optimization method are given in Section 5 and 6; the studied problem is formulated as convex in Section 7; heuristic decisions are described in Section 8; examples of battery sizing for a single and double buffer system are given in Section 9 and 10; and the paper is ended with discussion and conclusion in Section 11 and 12.

\section{Background on convex optimization}

This section gives a brief overview on convex optimization. The notation dom $f$ meaning domain of $f$ is adopted from [21]. The sets of real numbers is denoted by $\mathbb{R}$.

Definition 1. The set $C \subseteq \mathbb{R}^{n}$ is convex if the line segment between any two points $x, y \in C$ lies in $C$, i.e. $\theta x+(1-\theta) y \in C$ for any $\theta$ with $0 \leq \theta \leq 1$.
Definition 2. A function $f: \mathbb{R}^{n} \rightarrow \mathbb{R}$ is convex if $\operatorname{dom} f$ is a convex set and $f(\theta x+(1-\theta) y) \leq \theta f(x)+(1-\theta) f(y)$ for all $x, y \in \operatorname{dom} f$ and any $\theta$ with $0 \leq \theta \leq 1$.

The function $f$ is said to be concave if $-f$ is convex. The following remarks follow directly from Definition 2 .

Remark 1. An affine function $f(x)=q x+r$ is both convex and concave.

Remark 2. A quadratic function $f(x)=p x^{2}+q x+r$ with $\operatorname{dom} f \subseteq \mathbb{R}$ is convex if $p \geq 0$.

Remark 3. A quadratic-over-linear function $f(x, y)=x^{2} / y$ with $\operatorname{dom} f=\left\{(x, y) \in \mathbb{R}^{2} \mid y>0\right\}$ is convex.

Remark 4. A product $f(x, y)=x y$ is generally not a convex function.

Definition 3. The problem

$$
\begin{array}{cl}
\operatorname{minimize} & f_{0}(x) \\
\text { subject to } & f_{i}(x) \leq 0, \quad i=1, \ldots, m \\
& h_{j}(x)=0, \quad j=1, \ldots, p \\
& x \in \mathcal{X}
\end{array}
$$

is convex if $\mathcal{X} \subseteq \mathbb{R}^{n}$ is convex, $f_{i}(x), i=0, \ldots, m$ are convex and $h_{j}(x), j=1, \ldots, p$ are affine.

The following theorems, which proof can be found in [21], describe some of the operations that preserve convexity of sets and functions.

Theorem 1. An intersection $\mathcal{S}=\bigcap \mathcal{S}_{i}$, of convex sets $\mathcal{S}_{i}$, is a convex set.

Theorem 2. A nonnegative weighted sum $f=\sum w_{i} f_{i}$ with $w_{i} \geq$ 0 , of convex functions $f_{i}$, is a convex function.

This property can be extended to infinite sums and integrals. For example if $f(x, y)$ is convex in $x$ for each $y \in \mathcal{A}$, then $g(x)=$ $\int_{\mathcal{A}} w(y) f(x, y) d y$ is convex in $x$ if $w(y) \geq 0$.

Theorem 3. A pointwise maximum $f(x)=$ $\max \left\{f_{1}(x), \ldots, f_{m}(x)\right\}$, of convex functions $f_{i}(x), i=1, \ldots, m$, is a convex function.

\section{Bus line and charging infrastructure}

The driving cycle used to evaluate the PHEV powertrain originates from a real bus line in Gothenburg, see Fig. 1. The bus line is completely described by demanded velocity $v_{\text {dem }}(t)$ and road slope $\alpha(t)$ as functions of time. The reason Fig. 1 shows velocity and road altitude as functions of distance is for illustrative purposes.

It is assumed that there are $n_{c s}$ charging stations placed on bus stops that are on similar distance to each other. At the charging stations the bus can dock on or pick up electric power inductively to charge its battery.

The charging stations are generally classified into two groups: 1) conductive charging or wire coupling and 2) inductive charging or wireless coupling. Most conductive chargers 

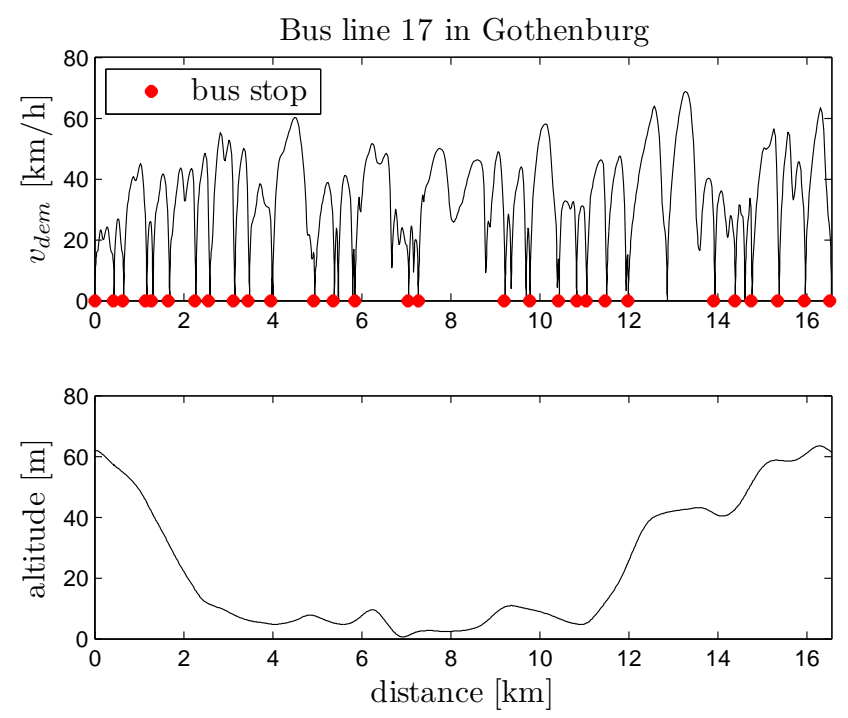

Figure 1: Driving cycle. The top plot shows demanded velocity and position of the bus stops. The bottom plot shows the road altitude.

presently deployed have efficiencies above $\eta_{c s}=92 \%$, and are able to maintain high efficiency at low power levels. Inductive chargers may have a peak efficiency of up to $92 \%$, but this efficiency drops off substantially with power level [24].

Significant drop in power, hence drop in efficiency at inductive chargers, takes place when batteries finish charging after being charged for long time periods [24]. For the problem in study the charging lasts for several seconds while the bus waits at the bus stop. Hence, it is reasonable to assume that the bus will charge mainly with high power at which constant average efficiency can be considered for both conductive and inductive chargers.

As a result, at the $j$ th charging station the bus can be charged with $\eta_{c s_{j}} P_{c s, \text { max }_{j}}$ usable power for the duration of the stop lasting $\Delta t_{c s_{j}}$ seconds. Hence, the electric power that the whole charging infrastructure provides can be expressed as

$$
\begin{aligned}
& P_{c s}(t)=\left\{\begin{array}{ll}
\eta_{c s_{j}} P_{c s, \max _{j}}, & t \in\left[t_{c s_{j}}, t_{c s_{j}}+\Delta t_{c s_{j}}\right] \\
0, & \text { otherwise }
\end{array},\right. \\
& j=0, \ldots, n_{c s}
\end{aligned}
$$

where $t_{c s_{j}}$ is the time when the bus arrives at the $j$ th charging station.

The electric power, $\boldsymbol{P}_{\text {grid }}(t)$, that the PHEV takes from the grid can be expressed as

$$
\begin{aligned}
& \boldsymbol{P}_{\text {grid }}(t)=\boldsymbol{x}_{c s}(t) P_{c s}(t) \\
& \boldsymbol{x}_{c s}(t) \in[0,1]
\end{aligned}
$$

where $\boldsymbol{x}_{\boldsymbol{c}}(t)$ is a decision variable that determines at which rate the energy buffer is to be charged when coupled to a charging station.

Note. For didactic reasons decision variables are marked in bold.

\section{PHEV powertrain model}

In this section, inverse simulation, quasi-stationary models are presented for both the series and the parallel powertrain. In the inverse simulation models the required power from the powertrain is determined by the driving cycle without using a driver model. This reduces the computational burden in simulations by removing vehicle velocity from the state vector [25]. The models are quasi-stationary, where inputs and outputs are related by static relations. This level of details is often used when deciding control strategies or comparing different vehicle concepts [17, 25-27].

\subsection{Series powertrain}

The system architecture for the series PHEV is shown in Fig. 2. The series powertrain includes an internal combustion engine (ICE), two electric machines and an energy buffer. The ICE together with the first electric machine (GEN) are used to supply electric energy to the energy buffer and to the second electric machine (EM) which is directly attached to the wheels. The ICE and GEN are not mechanically coupled to the wheels and are therefore considered as one unit, i.e. engine-generator unit (EGU).

The powertrain satisfies the power balance equations

$$
\begin{aligned}
& \boldsymbol{P}_{\text {dem }}(t)=\boldsymbol{T}_{\boldsymbol{E M}}(t) \omega_{E M}(t)+\boldsymbol{P}_{\boldsymbol{b r k}}(t) \\
& \boldsymbol{P}_{\boldsymbol{E M}, \boldsymbol{e l}}(t)=\boldsymbol{P}_{\boldsymbol{b}}(t)+\boldsymbol{P}_{\text {grid }}(t)+\boldsymbol{P}_{\boldsymbol{E G U}}(t)-P_{\text {aux }}
\end{aligned}
$$

where $\boldsymbol{P}_{\text {dem }}(t)$, detailed below in (5), is power required by the driving cycle, $\boldsymbol{P}_{\boldsymbol{b r k}}(t)$ is power dissipated at the friction brakes, $\omega_{E M}(t), \boldsymbol{T}_{\boldsymbol{E M}}(t)$, and $\boldsymbol{P}_{\boldsymbol{E M}, \boldsymbol{e l}}(t)$ are speed, torque and electrical power of the EM, $\boldsymbol{P}_{\boldsymbol{b}}(t)$ is power of the energy buffer and $\boldsymbol{P}_{\boldsymbol{E G U}}(t)$ is electric power delivered by the EGU. For simplicity, the power used by the auxiliary devices, $P_{a u x}$, is assumed constant.

The energy buffer can be composed of any number of batteries

$$
\boldsymbol{P}_{\boldsymbol{b}}(t)=\sum_{j} \boldsymbol{P}_{\boldsymbol{b}_{j}}(t)
$$

but typically not more than two batteries are used, where one has high power density and the other high energy density. The batteries are constructed by connecting cells in parallel and series. Detailed model for the battery is further explained in Section 4.4 .

The demanded power

$$
\begin{aligned}
\boldsymbol{P}_{\text {dem }}(t) & =\boldsymbol{F}_{\text {dem }}(t) v_{d e m}(t) \\
\boldsymbol{F}_{\text {dem }}(t) & =\frac{I \dot{v}_{d e m}(t)}{R_{w}^{2}}+\boldsymbol{m}(t) \dot{v}_{d e m}(t)+\frac{\rho_{\text {air }}}{2} A_{f} c_{d} v_{d e m}^{2}(t) \\
& +\boldsymbol{m}(t) g c_{r} \cos \alpha(t)+\boldsymbol{m}(t) g \sin \alpha(t)
\end{aligned}
$$

is uniquely determined by the driving cycle, where $\boldsymbol{F}_{\boldsymbol{d e m}}(t)$ is demanded force, $A_{f}$ is vehicle frontal area, $c_{d}$ is drag coefficient, $c_{r}$ is rolling resistance coefficient, $\rho_{\text {air }}$ is air density, $g$ is gravitational acceleration and $R_{w}$ is wheel radius. The vehicle rotational inertia, $I$, is cumulative inertia seen at the wheels and 


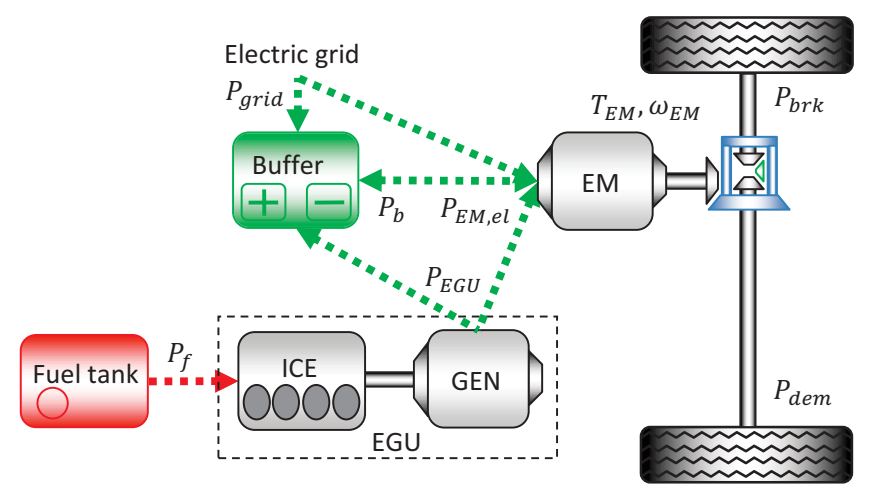

Figure 2: Series PHEV powertrain model.

includes the inertia of the wheels, the differential and the EM. The model does not account for the EGU inertia.

The total vehicle mass $\boldsymbol{m}(t)$ can be found as

$$
\boldsymbol{m}(t)=m_{v e h}(t)+\sum_{j} \boldsymbol{n}_{b c_{j}} m_{b c_{j}}
$$

where $\boldsymbol{n}_{\boldsymbol{b} \boldsymbol{c}_{j}}$ is number of cells and $m_{b c_{j}}$ is mass of each of the cells in the $j$ th battery. The mass of the vehicle without the batteries, $m_{v e h}(t)$, can vary in time due to the varying number of on-board passengers between bus stops.

\subsection{Parallel powertrain}

The system architecture for the parallel PHEV is shown in Fig. 3. The parallel powertrain includes an ICE and an EM mounted on the same shaft, delivering power to the wheels via a transmission unit. The powertrain satisfies the power balance equations

$$
\begin{aligned}
& \boldsymbol{P}_{\text {dem }}(\boldsymbol{t})=\boldsymbol{F}_{\gamma}(t) v_{d e m}(t)+\boldsymbol{P}_{\boldsymbol{b r k}}(t) \\
& \boldsymbol{T}_{\boldsymbol{s}}(t) \omega_{s}(t)=\boldsymbol{T}_{\boldsymbol{E} M}(t) \omega_{E M}(t)+\boldsymbol{T}_{\boldsymbol{I C E}}(t) \omega_{I C E}(t) \\
& \boldsymbol{P}_{\boldsymbol{E} \boldsymbol{M}, \boldsymbol{e l}}(t)=\boldsymbol{P}_{\boldsymbol{b}}(t)+\boldsymbol{P}_{\text {grid }}(t)-P_{\text {aux }}
\end{aligned}
$$

where $\boldsymbol{F}_{\gamma}(t)$ is force at the wheels generated by the ICE and/or $\mathrm{EM}, \boldsymbol{T}_{s}(t)$ and $\omega_{s}(t)$ are the torque and speed of the crankshaft, $\boldsymbol{T}_{\boldsymbol{E M}}(t), \omega_{E M}(t)$ and $\boldsymbol{P}_{\boldsymbol{E M}, \boldsymbol{e l}}(t)$ are the torque, speed and electrical power of the EM, and $\boldsymbol{T}_{\boldsymbol{I C E}}(t)$ and $\omega_{I C E}(t)$ are torque and speed of the ICE.

The demanded power and the rest of the variables in (7) are as described in Section 4.1. The vehicle rotational inertia includes the inertia of the wheels, the differential, the EM, the ICE and the transmission. It is time varying, as it depends on the choice of gear.

\subsection{Transmission}

The transmission is given by a static model

$$
\begin{aligned}
& C(\gamma(t)) \boldsymbol{F}_{\gamma}(t)= \begin{cases}\eta_{\gamma}(\gamma(t)) \boldsymbol{T}_{s}(t), & \boldsymbol{F}_{\gamma}(t) \geq 0 \\
\frac{1}{\eta_{\gamma}(\gamma(t))} \boldsymbol{T}_{s}(t), & \boldsymbol{F}_{\gamma}(t)<0\end{cases} \\
& \frac{1}{C(\gamma(t))} v_{d e m}(t)=\omega_{s}(t), \quad \gamma(t) \in\left\{0,1, \ldots, \gamma_{\max }\right\}
\end{aligned}
$$

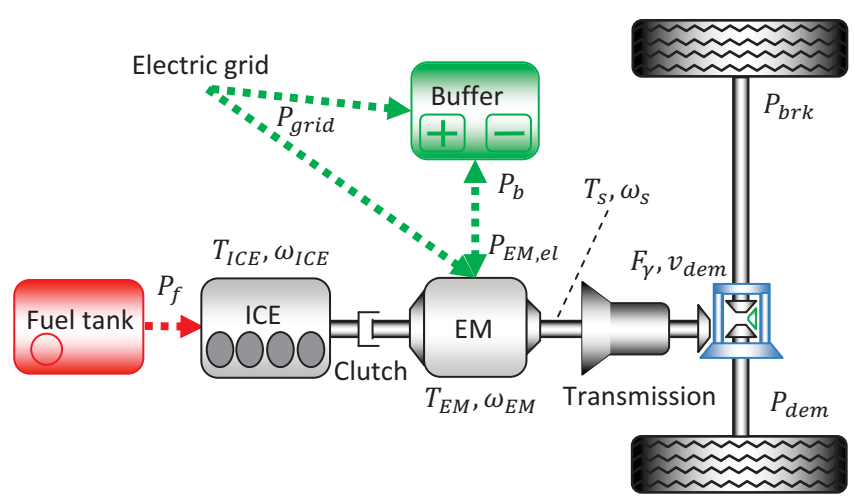

Figure 3: Parallel PHEV powertrain model.

where $C(\gamma(t))=R_{w} /\left(r_{d g} r_{\gamma}(\gamma(t))\right)$, and $\eta_{\gamma}(\gamma(t))$ and $r_{\gamma}(\gamma(t))$ are constant efficiency and gear ratio for gear $\gamma(t)$. The transmission consists of $\gamma_{\max }$ gears in addition to a final differential gear with ratio $r_{d g}$.

Assumption 1. The gear $\gamma(t)$ is determined prior to the optimization.

Based on the demanded vehicle velocity and traction force, heuristic rules are used to decide gear that permits the ICE and EM to operate with high efficiency. This is discussed in detail in Section 8.2.

\subsection{Battery}

The battery pack consists of $\boldsymbol{n}_{\boldsymbol{p}}$ strings connected in parallel with each string consisting of $\boldsymbol{n}_{\boldsymbol{s}}$ identical battery cells connected in series, see Fig. 4. The power of the battery pack with the cells modeled as simple resistive circuits [1], can be computed as

$$
\begin{aligned}
& \boldsymbol{P}_{\boldsymbol{b}}(t)=\left(V_{o c}(\boldsymbol{s o c}(t))-R \boldsymbol{i}(t)\right) \boldsymbol{i}(t) \boldsymbol{n}_{\boldsymbol{b c}} \\
& \boldsymbol{n}_{\boldsymbol{b c}} \geq 0
\end{aligned}
$$

where $\boldsymbol{i}(t)$ is the current in each of the strings, $V_{o c}(\boldsymbol{s o c}(t))$ is the open circuit voltage of the battery cell, $R$ is the cell resistance and $\boldsymbol{n}_{\boldsymbol{b c}}=\boldsymbol{n}_{\boldsymbol{s}} \boldsymbol{n}_{\boldsymbol{p}}$ is the total number of cells. The state of charge (SOC) of the battery cell is defined as

$$
\boldsymbol{\operatorname { s o c }}(t)=\operatorname{soc}_{0}-\frac{1}{Q} \int_{t_{0}}^{t} i(\tau) d \tau
$$

where $Q$ denotes the battery cell capacity and $s o c_{0}$ is the SOC at the initial time $t_{0}$. The SOC and cell current are limited by

$$
\begin{aligned}
& \boldsymbol{\operatorname { s o c }}(t) \in\left[\operatorname{soc}_{\text {min }}, \operatorname{soc}_{\text {max }}\right] \\
& \boldsymbol{i}(t) \in\left[i_{\text {min }}, i_{\text {max }}\right]
\end{aligned}
$$

where (11a) is imposed to extend the battery cycle and calendar life.

Additional possible constraints are starting at desired SOC value, preserving charge sustain operation, or limiting the Ah- 

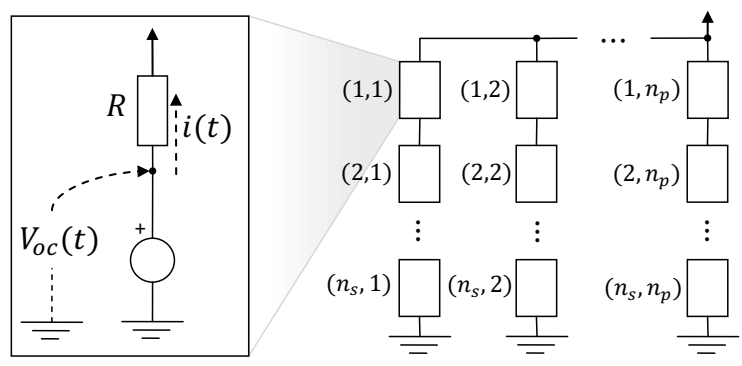

Figure 4: Equivalent battery circuit. The model of the battery cell is illustrated in the left side. The battery pack, illustrated in the right side, consists of $\boldsymbol{n}_{\boldsymbol{p}}$ strings connected in parallel, with each string containing $\boldsymbol{n}_{\boldsymbol{s}}$ identical cells connected in series.

throughput of the cell

$$
\begin{aligned}
& \operatorname{soc}\left(t_{0}\right)=\operatorname{soc}_{0} \\
& \operatorname{soc}\left(t_{0}\right)=\operatorname{soc}\left(t_{f l}\right) \\
& \int_{t_{0}}^{t_{f l}}|\boldsymbol{i}(t)| d t \leq Q_{A h} .
\end{aligned}
$$

The Ah-throughput associated to the driven cycle can be obtained from the maximum allowed Ah-throughput $Q_{A h \text {,max }}$ in the entire battery life of $y_{b}$ years, as

$$
Q_{A h}=Q_{A h, \max } \frac{\int_{t_{0}}^{t_{f l}} v_{d e m}(t) d t}{y_{b} s}
$$

where $s$ is the average distance traveled by the bus in one year.

\subsection{Engine-generator unit (EGU)}

The EGU is represented by a static model relating the fuel power $\boldsymbol{P}_{f}(t)$ to the electric power

$$
\tilde{\boldsymbol{P}}_{E G U}(t)=\eta_{E G U}\left(\boldsymbol{P}_{f}(t)\right) \boldsymbol{P}_{f}(t)
$$

by an efficiency model

$$
\begin{aligned}
\eta_{E G U}\left(\boldsymbol{P}_{f}(t)\right) & =\eta_{1}\left(1-e^{-\beta_{1}\left(\boldsymbol{P}_{f}(t)-P_{i d l e}\right)}\right) \\
& +\eta_{2} e^{-\beta_{2}\left(\boldsymbol{P}_{f}(t)-P_{f}^{*}\right)^{2}}
\end{aligned}
$$

that captures the essential EGU characteristics and compares reasonably well to manufacturer data [28]. Due to internal friction, the efficiency approaches zero at power lower than $P_{\text {idle }}$. Then, as $\boldsymbol{P}_{f}(t)$ increases, the efficiency increases with rate $\beta_{1}$ to a value close to $\eta_{1}$, see Fig. 5. The maximum EGU efficiency is about $\eta_{1}+\eta_{2}$ centered around $P_{f}^{*}$. The parameter $\beta_{2}$ determines the bulginess of the efficiency peak. Low $\beta_{2}$ value gives flatter curve around $P_{f}^{*}$, while higher $\beta_{2}$ gives a prominent peak.

The electric power is limited by

$$
\tilde{\boldsymbol{P}}_{\boldsymbol{E G U}}(t) \in\left[0, P_{E G U, \max }\right]
$$

and no electric power is generated when the ICE is idling or off. During cranking (starting-up the ICE before fuel has been injected) the ICE is modeled with a constant friction which in
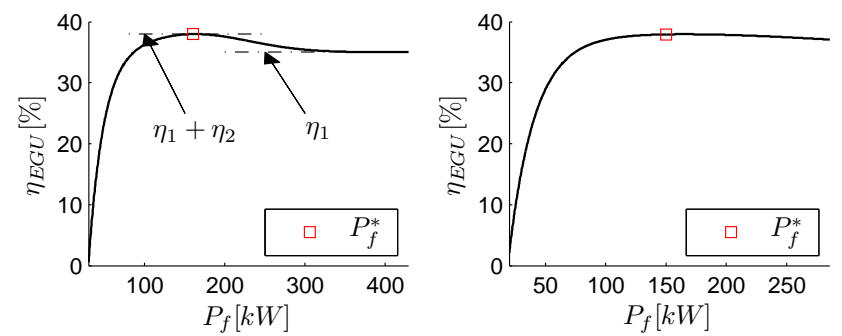

Figure 5: Model of two different EGUs. The EGU in the left plot has high $\beta_{2}$ value, while the EGU in the right plot has low $\beta_{2}$ value.

turn requires certain electric power $P_{\text {fric }} \leq 0$ from the GEN. Thus, the total EGU electric power is

$$
\boldsymbol{P}_{\boldsymbol{E G U}}(t)=e_{o n}(t) \tilde{\boldsymbol{P}}_{\boldsymbol{E G U}}(t)+e_{u p}(t) P_{f r i c}
$$

where the ICE on/off $e_{o n}(t)$ and start-up state $e_{u p}(t)$ are binary signals. The state $e_{o n}(t)$ in (16) also ensures that no power is generated when the ICE is off.

A simple decision for $e_{u p}(t)$ is to crank the ICE for $\Delta t_{u p}$ seconds before turning it on (injecting fuel). With a slight abuse of notation, by considering the binary as Boolean variables and reading 1 as true and 0 as false, $e_{u p}(t)$ can be obtained from

$$
e_{u p}(t)=e_{o n}\left(t+\Delta t_{u p}\right) \wedge \neg e_{o n}(t)
$$

This requires a constraint that prevents more than one change in $e_{o n}(t)$ in less than $\Delta t_{u p}$ seconds, i.e. the logical expression

$$
\neg\left(e_{o n}\left(t_{1}\right) \neq e_{o n}^{+}\left(t_{1}\right) \wedge e_{o n}\left(t_{2}\right) \neq e_{o n}^{+}\left(t_{2}\right)\right)
$$

needs to be true for all $t_{1}, t_{2} \in\left[t_{0}, t_{f l}\right] \wedge t_{1} \neq t_{2} \wedge\left|t_{2}-t_{1}\right|<\Delta t_{u p}$. The notation $e_{o n}^{+}(t)$ in (18) is the on/off state at the next time instant.

Assumption 2. The ICE on/off state is determined prior to the optimization.

The on/off state is found as any heuristic function of the speed profile, topography, distance to a charging station, time, etc., but the parameters governing the heuristics are kept constant during the optimization. A specific heuristic rule is discussed later in Section 8.

\subsection{Internal combustion engine (ICE)}

The ICE model relates the consumed fuel power to the delivered mechanical power

$$
\boldsymbol{P}_{f}(t)=\frac{\omega_{I C E}(t) \tilde{\boldsymbol{T}}_{\text {ICE }}(t)}{\eta_{I C E}\left(\omega_{I C E}(t), \tilde{\boldsymbol{T}}_{I C E}(t)\right)}
$$

by a static efficiency map $\eta_{I C E}\left(\omega_{I C E}(t), \tilde{\boldsymbol{T}}_{I C E}(t)\right)$, see the left plot of Fig. 6.

The ICE torque is limited by a torque limit dependent on the ICE speed

$$
\tilde{\boldsymbol{T}}_{\text {ICE }}(t) \in\left[0, T_{I C E, \max }\left(\omega_{I C E}(t)\right)\right]
$$



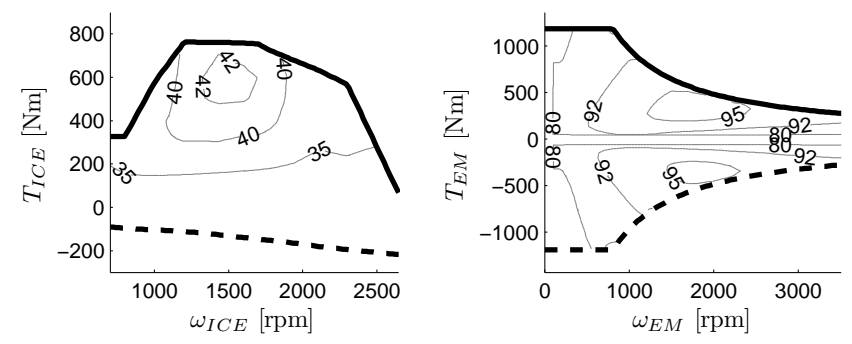

Figure 6: Model of an ICE (left) and an EM (right). The thin lines represent the efficiency of the components, the thick solid line is the maximum motoring torque and the dashed line is friction torque of the ICE and maximum generating torque of the EM, respectively.

where it is assumed that no mechanical power is generated when the ICE is idling or off. During cranking the ICE is modeled by a speed dependent friction torque $T_{\text {fric }}\left(\omega_{\text {ICE }}(t)\right) \leq 0$, yielding the total ICE torque

$$
\boldsymbol{T}_{I C E}(t)=e_{o n}(t) \tilde{\boldsymbol{T}}_{I C E}(t)+e_{u p}(t) T_{\text {fric }}\left(\omega_{I C E}(t)\right)
$$

with on/off and start-up state decided as in Section 4.5.

Under Assumption 1 that gear is decided a priori, the ICE speed is directly obtained from $v_{d e m}(t)$ and it is not decided by the optimization. Hence, the speed constraint

$$
\omega_{I C E}(t) \in\left[0, \omega_{I C E, \max }\right]
$$

is applied prior to the convex optimization. In the following section it will be shown that similar constraint holds for the EM. If these two constraints are violated then the problem is infeasible and there is no need for optimization. More details can be found in Appendix A.

\subsection{Electric machine (EM)}

The EM model, part of both the parallel and the series powertrain, relates the electrical to mechanical power by a static power loss map, $P_{E M, l o s s}\left(\omega_{E M}(t), \boldsymbol{T}_{E M}(t)\right)$ (for illustrative purposes, Fig. 6 depicts the efficiency map). This yields

$$
\boldsymbol{P}_{E M, e l}(t)=\omega_{E M}(t) \boldsymbol{T}_{\boldsymbol{E M}}(t)+P_{E M, l o s s}\left(\omega_{E M}(t), \boldsymbol{T}_{\boldsymbol{E M}}(t)\right)
$$

The EM torque is limited by torque limits dependent on the EM speed

$$
\boldsymbol{T}_{\boldsymbol{E M}}(t) \in\left[T_{E M, \min }\left(\omega_{E M}(t)\right), T_{E M, \max }\left(\omega_{E M}(t)\right)\right]
$$

where $\omega_{E M}(t)$ is uniquely determined from the crankshaft speed

$$
\omega_{E M}(t)=r_{E M} \omega_{s}(t)
$$

given the ratio of the EM reduction gear $r_{E M}$. Since transmission gear is decided a priori, see Assumption 1, the EM speed is obtained directly from $v_{d e m}(t)$ and is not decided by the optimization. Therefore, the physical speed limit

$$
\omega_{E M}(t) \in\left[0, \omega_{E M, \max }\right]
$$

is applied prior to the optimization.

\section{Problem formulation}

The studied sizing problem is formulated to minimize a cost function $J$ consisting of cost for consumed fuel and electricity and cost for energy buffer

$$
\begin{aligned}
J & =\frac{c_{f}}{Q_{f}} \int_{t_{0}}^{t_{f l}} \boldsymbol{P}_{f}(t) d t+\frac{c_{e l}}{3.6 \times 10^{6}} \int_{t_{0}}^{t_{f l}} \frac{\boldsymbol{P}_{\text {grid }}(t)}{\eta_{c s}(t)} d t \\
& +\sum_{j} c_{b e_{j}} \boldsymbol{n}_{\boldsymbol{b} \boldsymbol{c}_{j}}
\end{aligned}
$$

where $c_{f}$ is fuel price [currency/l], $Q_{f}$ is energy content of the fuel $[\mathrm{J} / \mathrm{l}], c_{e l}$ is electricity price [currency/kWh], $t_{0}$ and $t_{f l}$ are initial and final time of the driving cycle, and $\eta_{c s}(t)$ is charging efficiency which at each time instance may differ if the infrastructure consists of different charging stations. The cost of the battery cells $c_{b e_{j}}$ [currency] is an estimate based on the actual market cost of the battery (including cost for materials, manufacturing, packaging and circuitry), normalized by driven distance.

The optimization is subject to the constraints (1-26) and involves both time dependent and time invariant variables. Time dependent variables common to both the series and the parallel powertrain are $\boldsymbol{P}_{\text {grid }}(t), \boldsymbol{x}_{\boldsymbol{c s}}(t), \boldsymbol{P}_{\text {dem }}(t), \boldsymbol{F}_{\text {dem }}(t), \boldsymbol{P}_{\text {brk }}(t)$, $\boldsymbol{T}_{\boldsymbol{E} M}(t), \boldsymbol{P}_{\boldsymbol{E M}, \boldsymbol{e l}}, \boldsymbol{P}_{\boldsymbol{b}}(t), \boldsymbol{P}_{\boldsymbol{b}_{\mathbf{1}}}(t), \boldsymbol{P}_{\boldsymbol{b}_{\mathbf{2}}}(t), \boldsymbol{i}_{\mathbf{1}}(t), \boldsymbol{i}_{\mathbf{2}}(t), \boldsymbol{s o c}_{\mathbf{1}}(t), \boldsymbol{s o c}_{\mathbf{2}}(t)$, $\boldsymbol{P}_{f}(t)$ and $\boldsymbol{m}(t)$; common time invariant variables are $\boldsymbol{n}_{\boldsymbol{b} \boldsymbol{c}_{\mathbf{1}}}$ and $\boldsymbol{n}_{\boldsymbol{b} \boldsymbol{c}_{2}}$. Additional variables for the series powertrain are $\boldsymbol{P}_{\boldsymbol{E} \boldsymbol{G U}}(t)$ and $\tilde{\boldsymbol{P}}_{\boldsymbol{E} \boldsymbol{G U}}(t)$, and for the parallel powertrain $\boldsymbol{F}_{\boldsymbol{\gamma}}(t)$, $\boldsymbol{T}_{\boldsymbol{S}}(t), \boldsymbol{T}_{\boldsymbol{I C E}}(t)$ and $\tilde{\boldsymbol{T}}_{\text {ICE }}(t)$.

Note. The high number of optimization variables is only for didactic purposes to easily explain the remodeling of the problem into a convex form, which is the topic of Section 7. The problem can be rewritten in a more compact form, with less variables and constraints, as in Table 1.

\section{Optimization method}

This section shows a general framework of the algorithm that will be used for solving the battery sizing problem.

The cost function (27) is a nonnegative weighted sum of optimization variables, hence convex by Theorem 2 . For the problem to be convex, recall Definition 3, the functions in the inequality constraints should also be convex and the equality constraints should be affine. The underlying series and parallel PHEV powertrains consist of components that introduce constraints which do not comply to this requirement.

Section 7 will describe the necessary approximations for a reformulation of the original problem into a convex optimization problem. The convex problem can then be solved using generally available solvers, such as SeDuMi [22], or SDPT3 [23]. The investigation of the constraints involving time dependent variables is carried out at each specific time instance $t$. Then, the feasible set of the problem obtained by intersecting convex sets for all the time instances $t \in\left[t_{0}, t_{f l}\right]$ is convex by Theorem 1 .

The ICE on/off and gear heuristics may require the convex problem to be solved in several nested loops. Then, the steps 


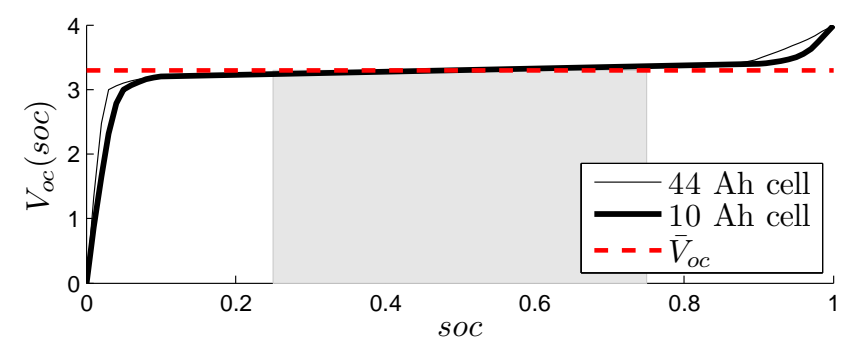

Figure 7: Model of the battery open circuit voltage. The solid lines represent the original models and the dashed line is the approximation. Good fit is expected in the allowed SOC range represented by the shaded region.

taken to solve the problem of battery sizing can be summarized as follows:

1. Define desired vehicle performance by deciding the driving cycle, as in Fig. 1.

2. Define a charging infrastructure by deciding on charging stations, their distribution on the driving cycle and charging interval per station.

3. Loop 1: Decide $e_{\text {on }}(t)$.

4. Loop 2 (for parallel powertrain only): Decide $\gamma(t)$.

5. In each iteration of the nested loops solve a convex problem.

\section{Convex modeling}

Starting from the convex cost function, this section augments the minimization problem by sequentially including the constraints from the powertrain components while describing approximations and remodeling of the constraints such that problem convexity is preserved.

\subsection{Battery}

The battery model described by the constraints (9-12) involves three time varying variables, $\boldsymbol{P}_{\boldsymbol{b}}(t), \boldsymbol{i}(t)$ and $\boldsymbol{s o c}(t)$, and one time invariant variable, $\boldsymbol{n}_{\boldsymbol{b} \boldsymbol{c}}$. The variable $\boldsymbol{P}_{\boldsymbol{b}}(t)$ is also involved in (4) as the power of one of the energy buffers in the double buffer system. By relaxing $\boldsymbol{n}_{\boldsymbol{b c}}$ to a real number, it is clear that from these constraints only (9a) may lead to a nonconvex problem, due to the non-convex voltage-SOC dependency, see Fig 7, and the product of two variables, recall Remark 4 .

To preserve the problem convexity, the following four steps are taken. First, the battery open circuit voltage is approximated as constant, i.e. $V_{o c}(\operatorname{soc}(t))=\bar{V}_{o c}$. Fig. 7 shows that the approximation gives good fit in the allowed SOC range for the $44 \mathrm{Ah}$ and $10 \mathrm{Ah}$ cells ${ }^{2}$ used in the examples in Section 9 and 10.

\footnotetext{
${ }^{2}$ The cells, high energy $44 \mathrm{Ah}$ and high power $10 \mathrm{Ah} \mathrm{LiFePO}_{4}$ lithiumion, are manufactured by Saft (http://www.saftbatteries.com, October 4, 2011) and can be found under the names Super-Phosphate VL 45E Fe and SuperPhosphate VL 10V Fe, respectively.
}

Second, the following variable changes

$$
\begin{aligned}
& \tilde{\boldsymbol{i}}(t)=\boldsymbol{n}_{\boldsymbol{b c}} \boldsymbol{i}(t) \\
& \boldsymbol{s} \tilde{\boldsymbol{o}} \boldsymbol{c}(t)=\boldsymbol{n}_{\boldsymbol{b c}} \boldsymbol{s o c}(t)
\end{aligned}
$$

are introduced; third, (9b) is restricted to permit strictly positive number of battery cells; and fourth, (9a) is relaxed with inequality. Then (9-12) can be rewritten as

$$
\begin{aligned}
& \boldsymbol{P}_{\boldsymbol{b}}(t) \leq \bar{V}_{o c} \tilde{\boldsymbol{i}}(t)-R \frac{\tilde{\boldsymbol{i}}^{2}(t)}{\boldsymbol{n}_{\boldsymbol{b c}}} \\
& \boldsymbol{n}_{\boldsymbol{b c}}>0 \\
& \boldsymbol{s} \tilde{\boldsymbol{o}} \boldsymbol{c}(t)=\boldsymbol{n}_{\boldsymbol{b c}} \operatorname{soc}_{0}-\frac{1}{Q} \int_{t_{0}}^{t} \tilde{\boldsymbol{i}}(\tau) d \tau \\
& \boldsymbol{s} \tilde{\boldsymbol{o}} \boldsymbol{c}(t) \in\left[\operatorname{soc}_{\min }, \operatorname{soc}_{\text {max }}\right] \boldsymbol{n}_{\boldsymbol{b c}} \\
& \tilde{\boldsymbol{i}}(t) \in\left[i_{\text {min }}, i_{\text {max }}\right] \boldsymbol{n}_{\boldsymbol{b c}} \\
& \boldsymbol{s} \tilde{\boldsymbol{o}} \boldsymbol{c}\left(t_{0}\right)=\operatorname{soc}_{0} \boldsymbol{n}_{\boldsymbol{b c}} \\
& \boldsymbol{s} \tilde{\boldsymbol{o}} \boldsymbol{c}\left(t_{0}\right)=\boldsymbol{s} \tilde{\boldsymbol{o}} \boldsymbol{c}\left(t_{f l}\right) \\
& \int_{t_{0}}^{t_{f l}}\left[\tilde{\boldsymbol{i}}(t) \mid d t \leq Q_{A h} n_{b c} .\right.
\end{aligned}
$$

These constraints preserve the problem convexity since they include only affine functions, except (28a) which has a nonlinear term. The nonlinearity is a quadratic-over-linear function, hence convex for $\boldsymbol{n}_{\boldsymbol{b c}}>0$, see Remark 3 .

The relaxation (28a) intuitively looks like a bad idea, since it means that there is a possibility of throwing electric energy away, which consequently will decrease the overall system efficiency. This is easier to observe if the constraint is rewritten as

$$
\begin{aligned}
& \boldsymbol{P}_{\boldsymbol{b}}(t)=\bar{V}_{o c} \tilde{\boldsymbol{i}}(t)-\boldsymbol{P}_{\boldsymbol{b}, \text { loss }}(t) \\
& \boldsymbol{P}_{\boldsymbol{b}, \text { loss }}(t) \geq R \frac{\tilde{\boldsymbol{i}}^{2}(t)}{\boldsymbol{n}_{\boldsymbol{b c}}}
\end{aligned}
$$

where $\boldsymbol{P}_{\boldsymbol{b}, \text { loss }}(t)$ is the power lost in the battery. It is easy to see, however, that the optimal solution will satisfy (28a) with equality, hence it will be optimal for the non-relaxed problem as well. For example, suppose that the optimal solution of the relaxed problem does throw power away at some time instant $t_{k}$, i.e. $\boldsymbol{P}_{\boldsymbol{b}, \text { loss }}\left(t_{k}\right)=R \tilde{\boldsymbol{i}}^{2}\left(t_{k}\right) / \boldsymbol{n}_{\boldsymbol{b c}}+\Delta P\left(t_{k}\right)$, with $\Delta P\left(t_{k}\right)>0$. Next, a new trajectory can be constructed where at the exact time instant the extra power $\Delta P\left(t_{k}\right)$ is not thrown away, and instead, it is used to power the wheels and hence reduce the ICE torque $\boldsymbol{T}_{\boldsymbol{I C E}}\left(t_{k}\right)$ or EGU power $\boldsymbol{P}_{\boldsymbol{E} \boldsymbol{G}}\left(t_{k}\right)$. This decreases the fuel power $\boldsymbol{P}_{f}\left(t_{k}\right)$, hence it decreases the cost (27), which shows that the solution could not have been optimal.

Note. With this problem formulation only the total number of battery cells can be obtained. For a more detailed battery design the number of cells in the string (or the number of strings) could then be uniquely determined by the terminal voltage of the whole pack.

Note. An integer solution for the number of battery cells, which is not part of this study, will need to rely on other optimization techniques (for a background, see, e.g. mixed integer nonlinear programming [29]). This, however, is a minor problem 
due to two reasons. First, if the battery is composed of smaller cells/modules that are not custom made for vehicle applications, then the battery may need many cells/modules and the rounding error has limited influence. Second, if the cells are custom made to be larger (or flat modules) the optimization gives an indication of what the total capacity should be. So in both cases there is not much need for an integer solution.

\subsection{Engine-generator unit (EGU)}

The EGU model described by (13-18) will lead to a nonconvex problem due to the non-convex efficiency model (13, 14). This can also be seen by graphically inspecting Fig. 8 .

The problem convexity can be preserved in two steps. First (14) is substituted in (13) and the resulting equation is approximated by a second order polynomial. Then the equality is relaxed to comply with the definition of a convex problem yielding

$$
\boldsymbol{P}_{f}(t) \geq\left(a_{0} \tilde{\boldsymbol{P}}_{\boldsymbol{E} \boldsymbol{G U}}^{\mathbf{U}}(t)+a_{1} \tilde{\boldsymbol{P}}_{\boldsymbol{E} \boldsymbol{G U}}(t)+P_{\text {idle }}\right) e_{o n}(t)
$$

where $a_{0} \geq 0$ and $a_{1}$ are found by least squares. The on/off state in (29) ensures that no fuel power is consumed when the ICE is off. The constraint (29) will preserve the problem convexity as its right hand side is convex by Remark 2 . It is easy to see, however, that at the optimum (29) will indeed hold with equality, since otherwise fuel will be wasted unnecessarily.

Models of two EGUs and their corresponding approximations are given in Fig. 8. The EGU model without prominent efficiency peak, see the right column of the figure, can be accurately approximated within the whole operating range. As the efficiency peak becomes bulgier, see the left column of the figure, the region in which the approximation error is small decreases and the approximation is not as good for high fuel powers. This, however, is not a major problem, because by minimizing fuel consumption the optimal solution will run the EGU mainly at high efficiency; hence, a bulgier efficiency peak will cause the ICE to operate closer to $P_{f}^{*}$ and mainly in the region where the approximation is valid. This is also confirmed by the example in Section 9.

\subsection{Transmission}

The transmission model described by constraint (8) involves two time dependent variables, $\boldsymbol{T}_{\boldsymbol{s}}(t)$ and $\boldsymbol{F}_{\gamma}(t)$, which are also part of $(7 \mathrm{a}, 7 \mathrm{~b})$. The constraints preserve the problem convexity, although recognizing this may not be obvious. For this reason, (8a) is rewritten as

$$
\boldsymbol{T}_{\boldsymbol{s}}(t) \geq \max \left\{\frac{C(\gamma(t)) \boldsymbol{F}_{\gamma}(t)}{\eta_{\gamma}(\gamma(t))}, C(\gamma(t)) \boldsymbol{F}_{\gamma}(t) \eta_{\gamma}(\gamma(t))\right\}
$$

where the pointwise maximum is convex by Theorem 3 and the constraint is relaxed with inequality to comply with Definition 3 .

At the optimum (30) will be satisfied with equality. Moreover, since $\eta_{\gamma}(\gamma(t)) \leq 1$ the shaft torque will be equal to the first input of the max function when demanded power is positive. Otherwise, the powertrain would produce more power than
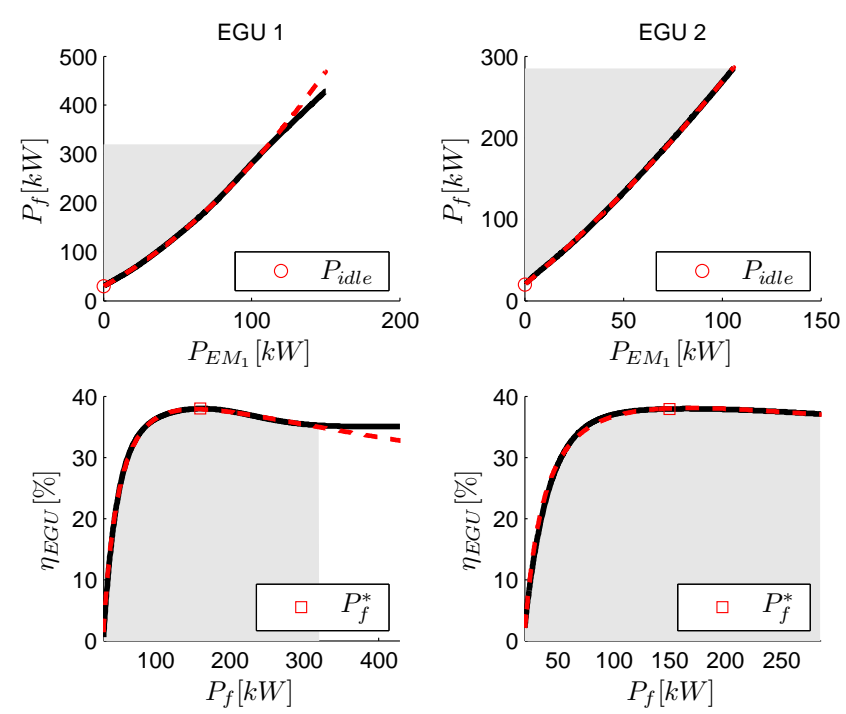

Figure 8: Original and approximated EGU models. The solid line represents the original function, while the dashed line is the approximation. The shaded region shows the interval in which good fit is to be expected.

needed which consequently would increase the total cost. Similarly, for negative power demands the shaft torque will be equal to the second input of the max function as otherwise the powertrain would not recuperate the available and free of charge braking energy.

Note. Some tools for solving convex problem, such as CVX [30], or YALMIP [31], directly recognize max as a convex function. Otherwise, an equivalent convex problem can be obtained by replacing (30) with inequalities where the left hand side is greater than or equal to all the inputs of the max function.

Note. With the same reasoning as above, it follows that the constraints (3a, 7a) for the series and parallel powertrain, respectively, can be relaxed to

$$
\begin{aligned}
& \boldsymbol{P}_{\text {dem }}(t) \leq \boldsymbol{T}_{\boldsymbol{E} M}(t) \omega_{E M}(t) \\
& \boldsymbol{P}_{\text {dem }}(t) \leq \boldsymbol{F}_{\gamma}(t) v_{d e m}(t)
\end{aligned}
$$

where the braking power has been taken out of the convex problem. Inequality in these equations, assuming the problem is feasible, will hold only when braking energy cannot be regenerated after the power limit of the generator (or a battery limit) has been met. This does not have any implication on the optimal results as the optimal braking power can still be obtained after the optimization has finished, from

$$
\begin{aligned}
& \boldsymbol{P}_{\boldsymbol{b r k}}^{*}(t)=\boldsymbol{P}_{\boldsymbol{d e m}}^{*}(t)-\boldsymbol{T}_{\boldsymbol{E} \boldsymbol{M}}^{*}(t) \omega_{E M}(t) \\
& \boldsymbol{P}_{\boldsymbol{b r k}}^{*}(t)=\boldsymbol{P}_{\boldsymbol{d e m}}^{*}(t)-\boldsymbol{F}_{\gamma}^{*}(t) v_{d e m}(t)
\end{aligned}
$$

where $*$ stands for the optimal value.

\subsection{Internal combustion engine (ICE)}

The ICE model described by (19-22) will lead to a nonconvex problem due to the non-convex efficiency map given in 


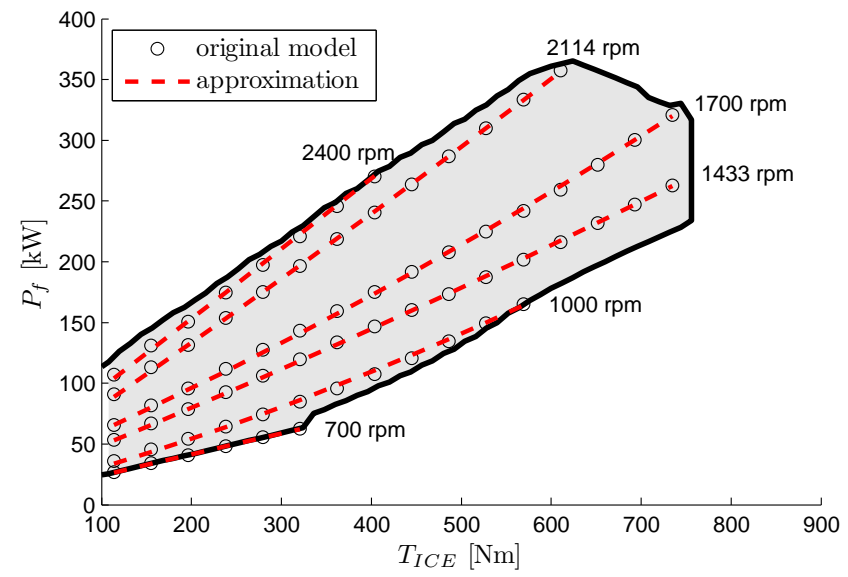

Figure 9: Fuel power of the original and the approximated ICE model for several ICE speeds. The shaded region, where good fit is to be expected, covers entirely the ICE operating range enclosed by solid lines.

(19). The problem convexity can be preserved by first approximating (19) with a second order polynomial and then relaxing it with inequality

$$
\begin{aligned}
\boldsymbol{P}_{f}(t) & \geq\left(c_{0}\left(\omega_{I C E}(t)\right) \tilde{\boldsymbol{T}}_{\boldsymbol{I C E}}^{\mathbf{2}}(t)\right. \\
& \left.+c_{1}\left(\omega_{I C E}(t)\right) \tilde{\boldsymbol{T}}_{I C E}(t)+c_{2}\left(\omega_{I C E}(t)\right)\right) e_{o n}(t)
\end{aligned}
$$

where the coefficients $c_{0}\left(\omega_{I C E}(t)\right) \geq 0, c_{1}\left(\omega_{I C E}(t)\right)$ and $c_{2}\left(\omega_{I C E}(t)\right)$ are found by least squares for a number of grid points of $\omega_{I C E}$. For speed values not belonging to the grid nodes, the coefficients are obtained by linear interpolation.

Similarly as with the EGU, it can be easily shown that at the optimum (31) will be satisfied with equality.

An example of the original and the approximated ICE model is illustrated in Fig. 9.

Note. Similar ICE representation, also known as Willans lines $[32,33]$, approximates the ICE model by affine relations. The approximation is a fairly accurate representation of actual engine data and has been verified on many different types of engines, from conventional spark ignition to compression ignition direct injection $[34,35]$.

\subsection{Electric machine (EM)}

The EM model described by (23-26) will lead to a nonconvex problem due to the non-convex power loss map in (23). The problem convexity can be preserved by approximating (23) with a second order polynomial and then relaxing it with inequality

$$
\begin{aligned}
\boldsymbol{P}_{\boldsymbol{E M}, \boldsymbol{e l}}(t) & \geq b_{0}\left(\omega_{E M}(t)\right) \boldsymbol{T}_{\boldsymbol{E M}}^{\mathbf{2}}(t) \\
& +b_{1}\left(\omega_{E M}(t)\right) \boldsymbol{T}_{\boldsymbol{E M}}(t)+b_{2}\left(\omega_{E M}(t)\right)
\end{aligned}
$$

where the coefficients $b_{0}\left(\omega_{E M}(t)\right) \geq 0, b_{1}\left(\omega_{E M}(t)\right)$ and $b_{2}\left(\omega_{E M}(t)\right)$ are found by least squares for a number of grid points of $\omega_{E M}$. For speed values not belonging to the grid nodes, the coefficients are obtained by linear interpolation.

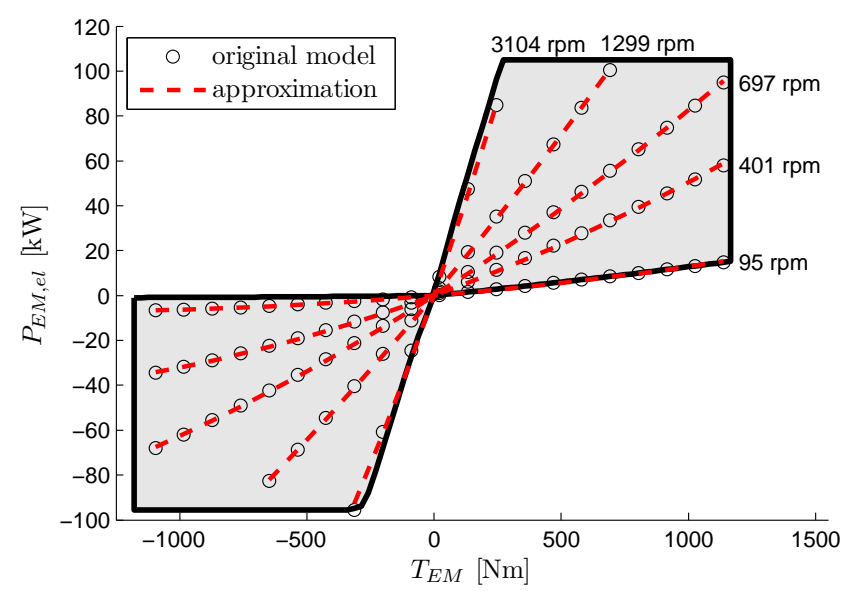

Figure 10: Electric power of the original and the approximated EM model for several EM speeds. The shaded region, where good fit is to be expected, covers entirely the EM operating range enclosed by solid lines.

It is easy to see that at the optimum (32) will hold with equality as otherwise electric energy will be thrown away unnecessarily.

The original and the approximated EM model are illustrated in Fig. 10.

\section{Heuristic decisions}

The engine on/off and transmission gear can be defined as any function independent on the variables decided by the convex optimization. This section describes heuristic decisions based on demanded power and speed.

\subsection{ICE on/off}

A typical control of HEVs when user comfort and drivability are not the main objectives is running the ICE in on/off mode. The strategy is common for both, the series [36, 37] and the parallel [38, 39] powertrains and relies on finding a threshold above which the ICE is turned on. In [39] a velocity threshold is used that minimizes fuel consumption on the studied driving cycle. In [38] the ICE is turned on when demanded power is above an optimal threshold and it has been shown that engine on/off improve fuel economy not only at low speeds and during decelerations, but also during highway cruising. Moreover, in [38] it is theoretically proven that this operation mode is indeed locally optimal using Pontryagin's minimum principle.

Likewise, the heuristic on/off decision used in this study turns on the engine when the demanded power $P_{\text {base }}(t)$ exceeds the threshold $P_{o n}^{*}$

$$
e_{o n}(t)= \begin{cases}1, & \text { if } P_{\text {base }}(t) \geq P_{o n}^{*} \\ 0, & \text { otherwise. }\end{cases}
$$

The power $P_{\text {base }}(t)$ is obtained by zero-phase moving average filtering of the demanded power of the so called "baseline" powertrain. For the series PHEV the baseline powertrain is the 
powertrain without batteries. For the parallel PHEV the baseline powertrain also excludes the inertia of the ICE, the EM and the transmission.

The power $P_{o n}^{*}$ is the optimal threshold that results in a feasible solution and minimizes the cost (27). It is obtained by first gridding the power $P_{\text {on, } i} \in\left[0 ; P_{\max }\right]$ and then solving the convex problem for each gridded value $P_{o n, i}$ as a switching threshold. The upper limit $P_{\max }$ is the maximum power the powertrain can deliver.

\subsection{Gear selection}

The heuristic gear selection used in this paper, and detailed in Appendix A, decides gear from the demanded power and speed of the powertrain with some initially guessed battery size. At each time instance gear is selected to optimize the EM efficiency when the ICE is off. If the ICE is on, the selected gear gives the optimal ICE efficiency of the feasible power split combinations that satisfy the demanded power.

In order to more accurately model the performance of, e.g. an Automated Manual Transmission [40] where during high torque transfer a gear shift might take up to 1-2 seconds to execute [41], two additional actions are taken: a moving average filter is used to remove too frequent gear shifts, see Appendix A; and no gear shifts are allowed during brake regeneration as the resulting torque interrupt would be too costly in terms of lost brake regeneration.

After the convex optimization has finished, the demanded power is recomputed with the recently obtained optimal battery size and the gear selection is repeated until no decrease in cost is detected. Experiments showed that the gear selection procedure usually ends in not more than three iterations even when the initial guess of battery size is far from the optimal.

\section{Example 1: Single energy buffer}

This section gives an example of optimal battery sizing of series and parallel plug-in hybrid electric bus. The purpose of the example is to investigate whether or not the solution of the convex problem will point toward a similar battery design as the solution of the non-convex problem which is to be solved by DP. By comparing the solutions conclusions can be drawn on the validity of the convex modeling approximations and heuristic decisions.

\subsection{Problem setup}

The vehicle includes one battery consisting of lithium-ion $44 \mathrm{Ah}$ identical cells with specifications listed in Table 2. It is assumed that cell packaging and circuitry account for $12.3 \%$ of the total mass of the battery pack [42]. The bus line, see Fig. 1, is equipped with seven charging stations installed on approximately equal distance to each other. All charging stations are Level 3 chargers (see [43] for background) with efficiency of $92 \%$ and provide $100 \mathrm{~kW}$ continuous power. It is also assumed, for simplicity, that the vehicle charging interval is $10 \mathrm{~s}$ at each station.
The battery cost of $c_{b}=178 € / \mathrm{kWh}$, an optimistic suggestion by [42], includes cost for materials, manufacturing, packaging and circuitry. Its payment is equally divided in $y_{p}=2$ years with $p=5 \%$ yearly interest rate, yielding total cost

$$
c_{b e}=c_{b} E_{b} m_{b c}\left(1+\frac{p}{100} \frac{y_{p}+1}{2}\right) \frac{\int_{t_{0}}^{t_{f l}} v_{d e m}(t) d t}{y_{p} s}
$$

where $E_{b}[\mathrm{kWh} / \mathrm{kg}]$ is specific energy determined from the entire energy content of the battery cell.

The series powertrain includes $150 \mathrm{~kW}$ EGU as in Fig. 13(a), and $180 \mathrm{~kW} 2400 \mathrm{rpm}$ EM which is a scaled version of the EM in Fig. 13(c). The parallel powertrain includes $130 \mathrm{~kW} \mathrm{ICE}$ and $100 \mathrm{~kW}$ EM as in Fig. 13(b) and 13(c), respectively, and 12 gear transmission. Both vehicles have identical chassis and total mass of $14 \mathrm{t}$ excluding the battery. It is assumed, for simplicity, that there is a constant number of passengers on board. The values for the rest of the parameters are given in Table 3.

The initial battery state is free, but charge sustaining operation is maintained, requiring the initial and final battery state to be equal. The assumption means that immediately after the final stop, the bus may start the route in opposite direction without waiting for more than the $10 \mathrm{~s}$ charging interval.

\subsection{The convex problem}

The convex problem written in a compact form, with lower number of variables and constraints, is summarized in Table 1. Before passing the problem to the solver, SeDuMi, the variables are rescaled and discretized with zero order hold. For a chosen sampling time $h$, the discretization will give $k=1, . ., N$ time instances. Hence each time dependent variable in the convex problem is actually a vector of $N=t_{f l} / h+1$ variables (with abuse of notation, the name of the discrete variables in Table 1 is kept the same as the name of the corresponding continuous variables). This will give large number of variables, e.g. solving the dual ${ }^{3}$ problem required calling SeDuMi with 28277 variables and 8438 equality constraints, for the series, and 28941 variables and 8224 equality constraints for the parallel powertrain.

The problem can also be rewritten in a conic form with a linear objective function and linear matrix inequalities, and it can therefore be recognized as a semidefinite program (SDP), see [21] for background. In this study, however, the problem is not manually transformed to the general SDP form, but a tool is used, CVX [30], to do this automatically. This allows writing the problem in its natural, more readable form, as in Table 1. To enhance readability even further, the problem in Table 1 is written using expressions, also supported by CVX. The expressions are described by symbols, e.g. $\boldsymbol{m}$ in Table 1 , which hold an operation over variables, e.g. $\boldsymbol{m}=m_{v e h}+\boldsymbol{n}_{\boldsymbol{b c}} m_{b c}$, with $\boldsymbol{n}_{\boldsymbol{b c}}$ being the variable. Before passing the convex problem to the solver, CVX replaces the expressions with the corresponding mathematical operations.

\footnotetext{
${ }^{3}$ For improved efficiency SeDuMi is solving the dual problem. The problem conversion from its standard form to a Lagrangian dual is done automatically by the solver. See [21] for background on duality.
} 
Table 1: Convex problem for a PHEV with a single battery.

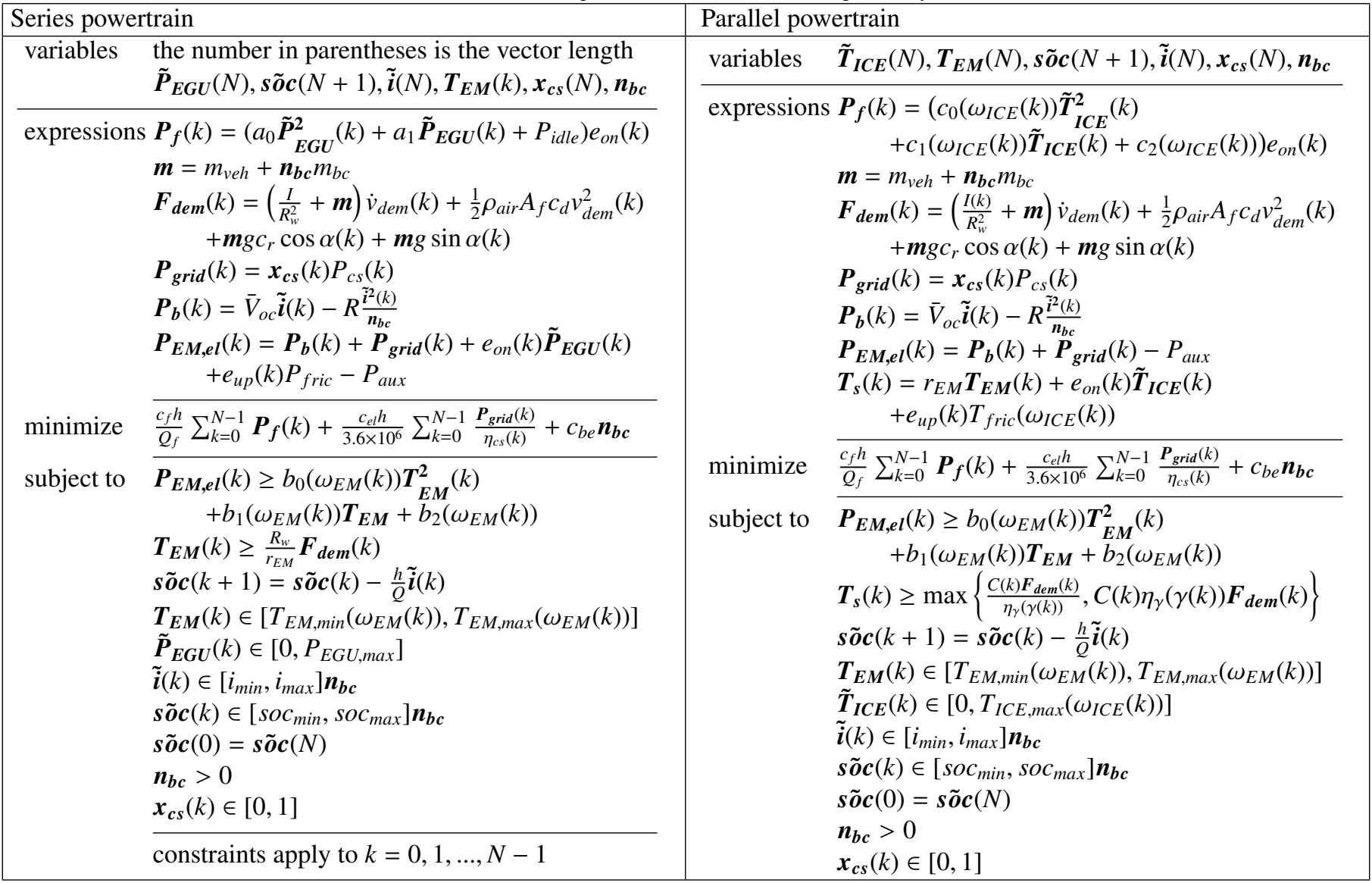

Note. The expressions are not equality constraints, but are just used to render the problem more readable.

\subsection{Results from the convex optimization}

The optimal operating points for both the series and the parallel powertrain are given in Fig. 13. It can be noticed in Fig. 13(a) that the EGU, which has been kept on for $23.4 \%$ of the time, is operated with high efficiency and mainly in the region where the approximated model fits well the original model. Similarly, the ICE and EM in the parallel powertrain are also operated with high efficiency which can be seen in Fig. 13(b) and 13(c). The parallel powertrain is driven mostly in high gear keeping the ICE on for $18.3 \%$ of the time and in gear not lower than 5, see Fig. 12.

The series powertrain consumes $19.271 / 100 \mathrm{~km}$ diesel, which is higher than the consumed $16.881 / 100 \mathrm{~km}$ by the parallel powertrain. Moreover, due to the bigger battery, 489 cells $(3.48 \%$ of the total vehicle mass) for the parallel and 394 cells $(2.82 \%$ of the total vehicle mass) for the series powertrain, the parallel powertrain can afford charging from the grid with in average $99.22 \%$ of the maximum charging rate and with only $22.8 \%$ non-recuperated braking energy. The average charging rate for the series powertrain is $98.4 \%$ with $26.6 \%$ non-recuperated braking energy.

With both powertrains, the battery SOC is relatively far from the limits, but the cell current is often at the limit of $\pm 50 \mathrm{~A}$,

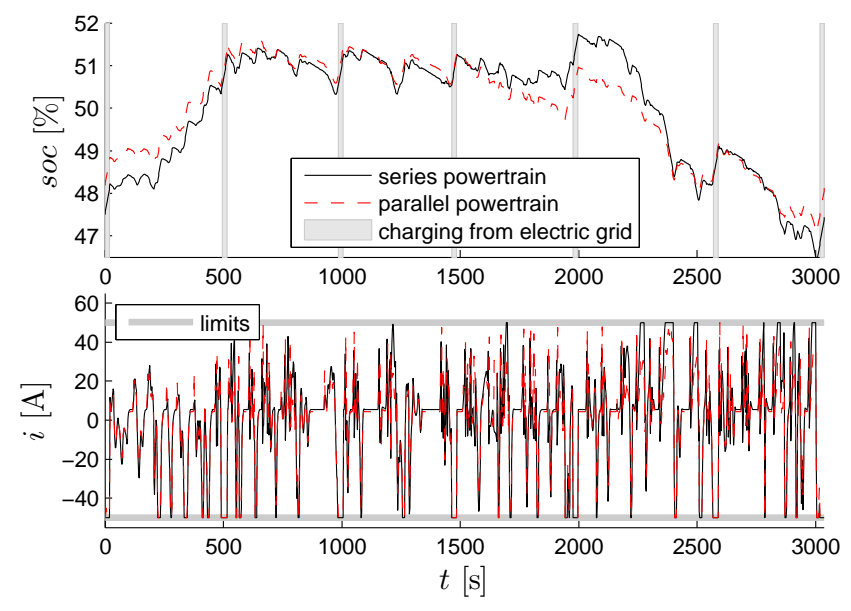

Figure 11: Battery state of charge and cell current along the bus line.

see Fig. 11. Moreover, since for a certain demanded power the cell current does not depend on the configuration of cells (series/parallel), recall (9), it can be concluded that the battery size is mainly influenced by its specific power. For this reason, in the example in Section 10 the powertrains are equipped with an additional battery with high specific power. 

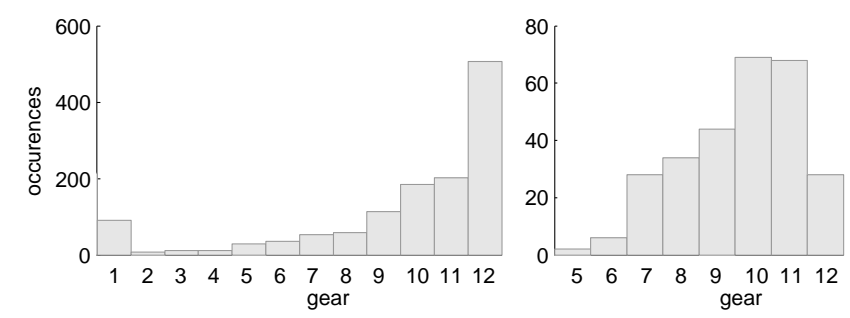

Figure 12: Gear distribution: left plot, from all time instances; right plot, only from times instances when the ICE is on.

\subsection{Dynamic programming (DP)}

DP uses Bellman's principle of optimality [14] to solve the problem via backwards recursion handling nonlinearities and constraints in a straightforward way. Denoting by $\boldsymbol{x}(t)$ and $\boldsymbol{u}(t)$ the states and control inputs, respectively, the problem of battery sizing is solved by running DP at gridded values of the battery size. For each grid value of $\boldsymbol{n}_{\boldsymbol{b c}}$ the algorithm finds the optimal power-split that minimizes the cost

$$
\begin{aligned}
& J_{D P}^{*}\left(\boldsymbol{x}\left(t_{k}\right), t_{k}\right)=\min _{\boldsymbol{u}\left(t_{k}\right)}\left\{J_{D P}^{*}\left(\boldsymbol{x}\left(t_{k+1}\right), t_{k+1}\right)+\delta\left(\boldsymbol{x}\left(t_{k}\right), \boldsymbol{x}\left(t_{k+1}\right)\right)\right. \\
& \left.+\frac{c_{f}}{Q_{f}} \int_{t_{k}}^{t_{k+1}} \boldsymbol{P}_{f}(t) d t+\frac{c_{e l}}{3.6 \times 10^{6}} \int_{t_{k}}^{t_{k+1}} \frac{\boldsymbol{P}_{\text {grid }}(t)}{\eta_{c s}(t)} d t\right\} \\
& t_{k} \in \mathcal{T}, \quad \boldsymbol{u}\left(t_{k}\right) \in \mathcal{U}, \quad \boldsymbol{x}\left(t_{k}\right) \in \mathcal{X}
\end{aligned}
$$

where $t_{k}$ and $t_{k+1}$ are consecutive time instances and $J_{D P}^{*}\left(\boldsymbol{x}\left(t_{k}\right), t_{k}\right)$ is a cost matrix holding the optimal cost-to-go from state $\boldsymbol{x}\left(t_{k}\right)$ at time $t_{k}$ to the desired final state at time $t_{f l}$. The function $\delta\left(\boldsymbol{x}\left(t_{k}\right), \boldsymbol{x}\left(t_{k+1}\right)\right)$ is used by the parallel powertrain to penalize gear shifts while braking.

The cost function (matrix) $J_{D P}^{*}$ is calculated over a grid of the time, the state and the control signals, i.e. the sets $\mathcal{T}, \mathcal{U}$ and $\mathcal{X}$ are discrete. The grid resolution determines the accuracy of the solution. For state values not belonging to $\mathcal{X}$, the cost is obtained by linear interpolation in $J_{D P}^{*}$.

The optimization is subject to the constraints described in Section 4. From these, the constraint for ending at the desired SOC state $s o c_{f l}$ is active only at the final time and it is formulated as a soft constraint

$$
J_{D P}^{*}\left(\boldsymbol{x}\left(t_{f l}\right), t_{f l}\right)=w_{s o c}\left(\boldsymbol{s o c}\left(t_{f l}\right)-\operatorname{soc}_{f l}\right)^{2}
$$

where deviations from the final state are penalized by a large positive number $w_{\text {soc }}$. The desired final state is chosen equal to the optimal final state obtained by the convex optimization.

To validate the solution of the convex optimization, DP is evaluated in three configurations which depending on the dimension of the state vector are named DP1, DP2 and DP3. DP1 has one state, $\boldsymbol{s} \boldsymbol{o c}\left(t_{k}\right)$, and is used to validate the approximations in the convex modeling for both the series and the parallel powertrain. The integer variables in this case are found by the same heuristics used by the convex optimization. DP2 and DP3 are used to simultaneously validate the convexifying approximations and the heuristic decisions for the series and the parallel powertrain, respectively. States in DP2 are $\boldsymbol{s o c}\left(t_{k}\right)$ and $\boldsymbol{e}_{\boldsymbol{o n}}\left(t_{k}\right)$, while DP3 additionally has gear, $\gamma\left(t_{k}\right)$, as a third state.

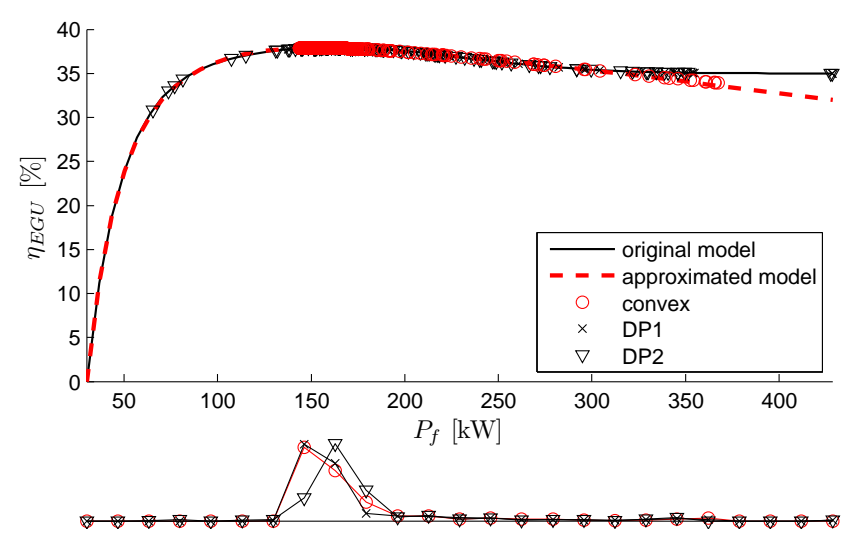

(a) EGU in the series powertrain.

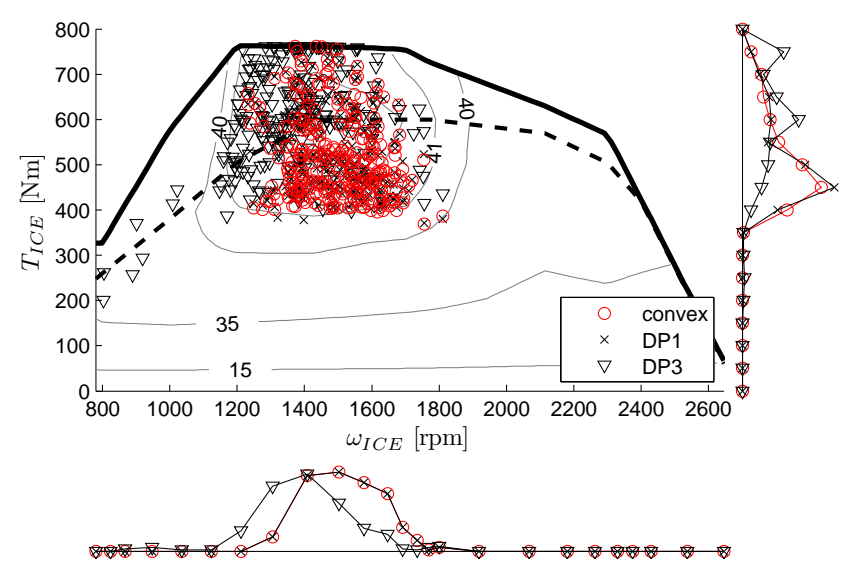

(b) ICE in the parallel powertrain. The dashed line is the optimal efficiency vs. engine speed.

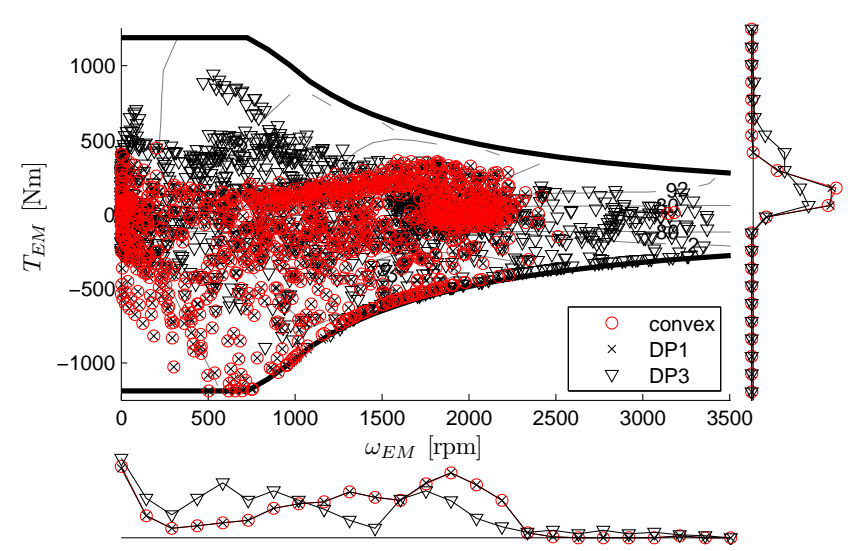

(c) EM in the parallel powertrain.

Figure 13: Optimal operating points from the convex optimization and DP. The distribution of the points, excluding zero power, is projected parallel to the axes.

The number of control inputs is decreased by substituting variables involved in equality constraints and by logical reasoning that the powertrain will try to recuperate as much braking power as possible and will try to charge from the grid with as high rate as possible. Control input in DP1 is $\boldsymbol{P}_{\boldsymbol{E G U}}\left(t_{k}\right)$ at the series and $\boldsymbol{T}_{\boldsymbol{I C E}}\left(t_{k}\right)$ at the parallel powertrain. DP2 and DP3 have an additional binary control for deciding the ICE on/off state, while DP3 also has a third control input for shifting gears. 


\subsection{DP vs. convex optimization}

The results from the convex optimization and DP are compared in Fig. 14. Although given in the same figure, these results are obtained in a different manner to better serve the validation purposes. The results from the convex optimization are obtained by varying the power threshold used in the engine on/off heuristics. For each threshold a unique on/off and gear decision is obtained which when used in the convex optimization will give cost and battery size, plotted in the figure, that are optimal for the used heuristics. The same on/off and gear heuristics are then employed in DP1, but using the original PHEV models without the convexifying approximations. The results show that the error due to the approximations is very small. Both algorithms indicate the same optimal battery size and similar fuel consumption, thus increasing the confidence that the approximations proposed in the convex modeling are acceptable for the PHEV powertrain sizing problem.

The results from DP2 and DP3 are obtained by varying the number of battery cells. Then, for each battery size DP finds the optimal on/off and gear that minimize fuel consumption. The results point to similar battery sizes to those obtained by convex optimization, but apparently there is difference in optimal fuel consumption. This is especially evident at the parallel powertrain where two heuristic choices had to be made and it shows that the heuristics have significant influence on the result and need to be improved.

It is important to note that the results of DP3 represent the best possible gear and on/off which are not easily obtained in reality. This is because the DP optimization exploits the fact that the future driving is perfectly known, although in reality there are driving uncertainness that heavily influence the decisions for the integer variables [44]. The gear heuristics proposed in this study decide gear at some time instance using information given only at that time instance. Hence, if the vehicle starts to brake at the next time instance it will need to stay at the previously decided gear. On the contrary, DP will decide the best gear before braking such that the powertrain efficiency is optimized during the upcoming braking period. As a consequence, DP is exploiting the EM more efficiently, e.g. by operating the EM at higher speed, see Fig. 13(c).

The optimal operating points obtained by DP are plotted in Fig. 13, together with the optimal points from the convex optimization. It can be noticed that due to the on/off heuristics the convex optimization avoids ICE (EGU) operation at low power. Fig. 13(a) also shows that the convex optimization avoids EGU operation at very high power, due to the approximation that lowered the EGU efficiency at the high power region. Nevertheless, the operating points from both the algorithms are mainly scattered in the same region where the ICE and the EGU are most efficient.

One execution of the convex algorithm required about $18.2 \mathrm{~s}$ for the series and $21.4 \mathrm{~s}$ for the parallel powertrain ${ }^{4}$. The computation time for one execution of DP1 was about $35 \mathrm{~min}$ for

\footnotetext{
${ }^{4}$ All investigations are carried out on the same computer with $2.67 \mathrm{GHz}$ dual core $\mathrm{CPU}$ and $4 \mathrm{~GB}$ RAM.
}
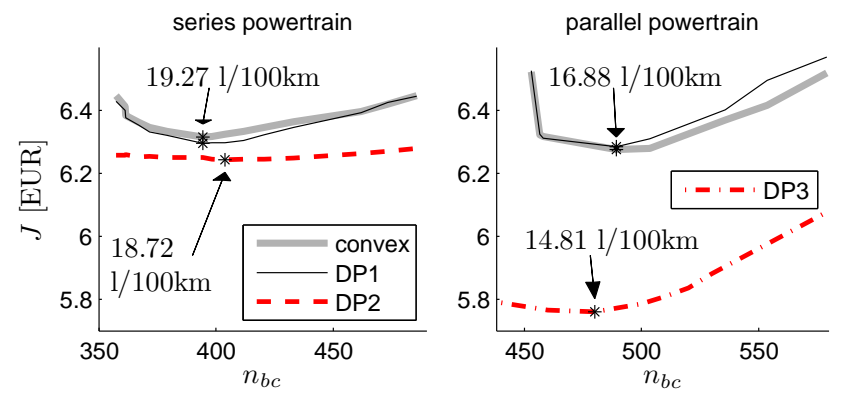

Figure 14: Cost vs. battery size obtained by convex optimization, and DP with one, two and three states, denoted by DP1, DP2 and DP3, respectively. The star indicates the optimal value.

both the powertrains. This long computation time is partly due to the solver which is generic and not dedicated to this type of problems. However, the main reason for the long computation time is the high grid resolution, 1000 grid points for SOC and 500 for the continuous inputs, needed to obtain accurate results (in some cases the cost changed very little with the number of battery cells, see the dashed line in Fig. 14). DP2 required even longer time, $1.7 \mathrm{~h}$, while DP3 was solved using a cluster of computers to keep computation time within reasonable limits.

Both algorithms need to be run several times, where the convex optimization iterates for finding the heuristic decisions and DP iterates on grid over the battery size. By using 50 grid points for the power threshold in the on/off heuristics, it was found that the whole procedure of battery sizing can be solved by convex optimization in $15.2 \mathrm{~min}$ for the series and $31 \mathrm{~min}$ for the parallel powertrain.

\section{Example 2: Double buffer system}

This section presents an example where the convex modeling approach enables an efficient solution of a sizing problem with two state variables and two sizing variables. The example studies how battery prices influence the battery sizes in a double buffer PHEV for fixed Level 2 and Level 3 infrastructure. The charging stations are all assumed to provide the same magnitude of power, which is $10 \mathrm{~kW}$ for Level 2 and $100 \mathrm{~kW}$ for Level 3 stations. The vehicle charging interval is $10 \mathrm{~s}$ at each station.

The vehicle setup for both the series and the parallel powertrain is as in Section 9, except that the powertrains are equipped with an additional Lithium-ion battery consisting of high-power $10 \mathrm{Ah}$ cells, see Table 2. The payment for both batteries is equally divided in 2 years with $5 \%$ yearly interest rate yielding total battery cost computed as in (34). The available SOC range for both batteries is $25-75 \%$.

We let the price for the $44 \mathrm{Ah}$ cell take values of 502, 178 and $107 € / \mathrm{kWh}$, where the highest price is the estimated sales price in year 2000, the second price is an optimistic cost, and the third price is the goal of the United States Advanced Battery Consortium for high-energy cells [42]. The price for the $10 \mathrm{Ah}$ 
cell is allowed to be 5,20 , or 30 times the price of the $44 \mathrm{Ah}$ cell.

\subsection{Optimization results}

The optimal number of battery cells is given in Fig. 15. The plots are organized in two rows and three columns, where the top row shows results for the series and the bottom row for the parallel powertrain. The three columns show results for the three different battery prices. From the results, the following conclusions can be drawn.

When the price of the high-power cells is 5 times the price of the high-energy cells, the high-power cells are dominant in the energy buffer. At a price ratio of 20 the buffer is made of similar number of the two types of cells, while at price ratio of 30, the high-energy cells become dominant. Yet, even the small number of high-power cells makes significant difference to the optimal number of high-energy cells. For example, the 489 high-energy cells found in Section 9 for the single buffer parallel powertrain will decrease to 357 by adding only 7 highpower cells (see solid line and triangle at the bottom row and middle column of Fig. 15). This number further decreases to 60 when there are 59 high-power cells in the buffer. In other words, the optimal buffer mass decreases from $504 \mathrm{~kg}$ of the single high-energy buffer to $102 \mathrm{~kg}$ of the double buffer with 60 high energy and 59 high-power cells.

The results also show that Level 3 infrastructure typically demands larger battery than Level 2 infrastructure when the $44 \mathrm{Ah}$ cells cost not more than $178 € / \mathrm{kWh}$. This is because the total cost for battery and used electricity is lower than the cost for used diesel, hence it is optimal to increase the battery size with the charging power to make room for storing the relatively cheap electric energy from the grid. The increase in number of cells, however, is very sensitive to the battery price and type. For example, the buffer size of the powertrain equipped with mainly high-power cells (see circles) is not significantly influenced by the charging power when the price is rather high, i.e. $5 \times 502 € / \mathrm{kWh}$. However, it is visibly sensitive to charging power for lower battery prices. On the contrary, the buffer size of a powertrain containing mainly high-energy cells is more sensitive to charging power for high battery prices (compare 2nd and 3rd column, triangles).

\section{Discussion}

This section points out the strengths and weaknesses of the proposed methodology and discusses possible enhancements of the model descriptions.

\subsection{Pros and cons of convex optimization and DP}

The main reason of using convex optimization is the relatively short computation time with regard to the problem dimensionality (number of states). Moreover, generic solvers and parsers exist, $[22,23,30,31]$, which allow the problems to be written in a very readable and easily extendable form. There is also vast amount of literature on both convex optimization and
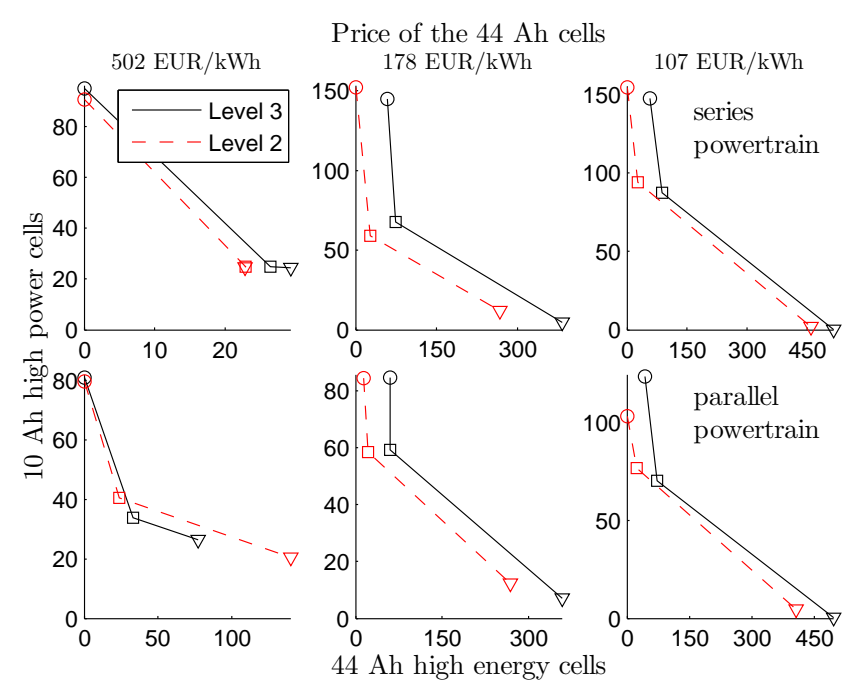

Figure 15: Optimal number of battery cells: top row, series powertrain; bottom row, parallel powertrain. The battery size at price ratio $10 \mathrm{Ah}$ cell/44 $\mathrm{Ah}$ cell $\in\{5,20,30\}$ is illustrated by \{circle, square, triangle\}, respectively.

on techniques to distribute the optimization over several computers [45]. This would allow, e.g. optimization of charging infrastructure in an entire city and simultaneous battery sizing of several buses driven on several bus lines.

This study showed that the problem of battery sizing can be considered as convex where for certain powertrain components the convexifying approximations have small influence on the optimal result. Using other powertrain components, such as super-capacitors, or batteries with visible voltage-SOC dependency, requires further studies as they cannot be used directly by the proposed methodology. Further studies are also needed to improve the heuristics for the integer variables since these can influence the optimal result more visibly. Nevertheless, the convex optimization may give significant contribution to problems which DP may have difficulties to solve, such as sizing the double buffer system.

For one-dimensional problems DP may still be the most attractive choice. Some of the reasons are that the problems can be non-convex, nonlinear, mixed-integer; DP problems can be easily parallelized on multiple processors; stochastic information can be easily included, but at the expense of increasing the size of dynamic states [46]. Furthermore, DP is widely used for $\mathrm{HEV}$ powertrain assessment and sizing, with high amount of academic literature and dedicated solvers to speed up computation time, [47, 48]. Yet, even for one-dimensional problems, some possibilities that are straightforward in the convex optimization are difficult to implement in DP, or will significantly decrease its performance. For example, having free final state in DP, but still maintaining charge sustain operation (as used in Example 1 when solved by convex optimization), will require running DP iteratively for each grided SOC as a final state value. Moreover, the constraint on the Ah-throughput of the battery (12) that is straightforward to include in the convex problem, will require an additional state in DP. 


\subsection{Enhanced models}

Although the proposed methodology was applied on fairly detailed models, there are possibilities to enhance the vehicle and infrastructure models without compromising the problem convexity. For example, the constant losses of the charging stations in (1) can be replaced with any nonlinear losses that are convex on charging power. Similarly, the losses of the power electronics that were not considered in this study, can be modeled as any convex function on $\tilde{\boldsymbol{i}}(t)$ by still preserving the problem convexity. Furthermore, the inertial effect of the EGU (in terms of increase in demanded power), which was not considered due to the EGU model that does not give information of its speed, can be included as long as it can be approximated as a convex function on the EGU power.

The proposed methodology can also be used to simultaneously maximize payload (amount of mass the vehicle can carry) as long as the cost can be expressed as a convex function on the carried mass.

Other more subtle modeling details that require further studies are simultaneous optimization of the ICE, EM, EGU sizing and the possibility to include thermal models of the powertrain components.

\section{Conclusion}

This paper presented a method for simultaneous optimization of power management control and battery sizing of series and parallel PHEV powertrains. The optimization problem is approximated by a nonlinear convex problem, using heuristics for the ICE on/off operation and gear selection.

This study also showed an example of battery sizing for a powertrain with a single battery and complete code was provided as it can be implemented in CVX. The result indicated that the convexifying approximations have small influence on the optimal solution, but higher attention is required to improve the on/off and gear heuristics.

An additional example solved the problem of battery sizing for a powertrain with two different batteries. The result showed that the sizing of the energy buffer is a delicate problem, where the optimal buffer size is not only sensitive to battery price, but also to charging power. This motivates further investigations for simultaneous optimization of powertrain sizing and charging infrastructure design.

\section{Appendix A. Gear selection}

The gear selection procedure is carried out in six steps:

1) The demanded force by the powertrain with some initially guessed number of battery cells, $F_{\text {base }}(t)$, is computed.

2) The shaft speed and torque for gear $\bar{\gamma}$ at time instant $t$ are
Table 2: Battery cells.

\begin{tabular}{|c|c|}
\hline \multicolumn{2}{|l|}{44 Ah cell } \\
\hline Nominal voltage & $\bar{V}_{o c}=3.3 \mathrm{~V}$ \\
\hline Specific energy & $E_{b}=155 \mathrm{Wh} / \mathrm{kg}$ \\
\hline Specific power & $0.18 \mathrm{~kW} / \mathrm{kg}$ \\
\hline Max. continuous current & $i_{\max }=i_{\min }=50 \mathrm{~A}$ \\
\hline Mass & $m_{b c}=0.9 \mathrm{~kg}$ \\
\hline \multicolumn{2}{|l|}{10 Ah cell } \\
\hline Nominal voltage & $\bar{V}_{o c}=3.3 \mathrm{~V}$ \\
\hline Specific energy & $E_{b}=55 \mathrm{Wh} / \mathrm{kg}$ \\
\hline Specific power & $5 \mathrm{~kW} / \mathrm{kg}$ \\
\hline Max. continuous discharge current & $i_{\max }=1750 \mathrm{~A}$ \\
\hline Max. charge current & $i_{\min }=300 \mathrm{~A}$ \\
\hline Mass & $m_{b c}=0.6 \mathrm{~kg}$ \\
\hline
\end{tabular}

found as

$$
\begin{aligned}
& \bar{\omega}_{s}(\bar{\gamma})=\frac{1}{C(\bar{\gamma})} v_{\text {dem }}(t) \\
& \bar{T}_{s}(\bar{\gamma})= \begin{cases}\frac{C(\bar{\gamma}) F_{\text {base }}(t)}{\eta_{\gamma}(\bar{\gamma})}-e_{\text {up }}(t) T_{\text {fric }}\left(\bar{\omega}_{I C E}(\bar{\gamma})\right), & F_{\text {base }}(t) \geq 0 \\
T_{s, \min }(\bar{\gamma}), & F_{\text {base }}(t)<0\end{cases} \\
& T_{s, \min }(\bar{\gamma})=\max \left\{C(\bar{\gamma}) \eta_{\gamma}(\bar{\gamma}) F_{\text {base }}(t)-e_{\text {up }}(t) T_{\text {fric }}\left(\bar{\omega}_{I C E}(\bar{\gamma})\right),\right. \\
& \left.r_{E M} T_{E M, \text { min }}\left(\bar{\omega}_{E M}(\bar{\gamma})\right)\right\}
\end{aligned}
$$

where it is reasonably assumed that the powertrain attempts to recuperate as much braking energy as possible. To simplify the derivations that follow, the ICE friction torque during start up is accounted within $\bar{T}_{s}(\bar{\gamma})$ above. The EM speed is $\bar{\omega}_{E M}(\bar{\gamma})=$ $r_{E M} \bar{\omega}_{s}(\bar{\gamma})$ and the ICE speed, when the ICE is on or at start up, is $\bar{\omega}_{I C E}(\bar{\gamma})=\bar{\omega}_{s}(\bar{\gamma})$. Then, the time-dependent set of feasible gears $\mathcal{G}_{t}$ at time instant $t$, is

$$
\begin{aligned}
\mathcal{G}_{t}= & \left\{\bar{\gamma} \mid \bar{\gamma} \in\left\{0,1, \ldots, \gamma_{\max }\right\}\right. \\
\wedge & \bar{\omega}_{E M}(\bar{\gamma}) \leq \omega_{E M, \text { max }} \\
\wedge & \neg\left(e_{o n}(t) \wedge \bar{\omega}_{I C E}(\bar{\gamma})<\omega_{\text {idle }}\right) \\
\wedge & \neg\left(e_{o n}(t) \wedge \bar{T}_{s}(\bar{\gamma})>T_{I C E, \max }\left(\bar{\omega}_{I C E}(\bar{\gamma})\right)\right. \\
& \left.\quad+r_{E M} T_{E M, \max }\left(\bar{\omega}_{E M}(\bar{\gamma})\right)\right) \\
\wedge & \left.\neg\left(\neg e_{o n}(t) \wedge \bar{T}_{s}(\bar{\gamma})>T_{E M, \max }\left(\bar{\omega}_{E M}(\bar{\gamma})\right)\right)\right\}
\end{aligned}
$$

where $\omega_{\text {idle }}$ is the idling speed, and it is assumed that the ICE and EM are sized for equal speed range, i.e. $\omega_{E M, \max } / r_{E M}=$ $\omega_{\text {ICE, max }}$.

3) For each $\bar{\gamma} \in \mathcal{G}_{t}$ a set of feasible ICE torques is obtained

$$
\begin{aligned}
\mathcal{T}_{I C E_{t \bar{\gamma}}}=\left\{\bar{T}_{I C E}(\bar{\gamma}) \mid e_{o n}(t)\right. \\
\quad \wedge \bar{T}_{I C E}(\bar{\gamma})+r_{E M} \bar{T}_{E M}(\bar{\gamma})=\bar{T}_{s}(\bar{\gamma}) \\
\wedge \bar{T}_{I C E}(\bar{\gamma}) \in\left[0, T_{I C E, \max }\left(\bar{\omega}_{I C E}(\bar{\gamma})\right)\right] \\
\left.\quad \wedge \bar{T}_{E M}(\bar{\gamma}) \in\left[T_{E M, \min }\left(\bar{\omega}_{E M}(\bar{\gamma})\right), T_{E M, \max }\left(\bar{\omega}_{E M}(\bar{\gamma})\right)\right]\right\}
\end{aligned}
$$


Table 3: Parameter values

\begin{tabular}{l|l||l|l}
\hline Parameter & Value & Parameter & Value \\
\hline$A_{f}$ & $8 \mathrm{~m}^{2}$ & $\eta_{\gamma=1: 5}$ & 0.97 \\
$c_{d}$ & 0.7 & $\eta_{\gamma=6: 11}$ & 0.98 \\
$c_{r}$ & 0.005 & $\eta_{\gamma=12}$ & 0.99 \\
$R_{w}$ & $0.47 \mathrm{~m}$ & $\rho_{\text {air }}$ & $1.293 \mathrm{~kg} / \mathrm{m}^{3}$ \\
$I_{E M}(100 \mathrm{~kW})$ & $1.3 \mathrm{kgm}^{2}$ & $g$ & $9.82 \mathrm{~m} / \mathrm{s}^{2}$ \\
$I_{E M}(180 \mathrm{~kW})$ & $2.1 \mathrm{kgm}^{2}$ & $h$ & $2 \mathrm{~s}$ \\
$I_{I C E}$ & $2.1 \mathrm{kgm}^{2}$ & $N$ & 1520 \\
$I_{w}$ & $10 \mathrm{kgm}^{2}$ & $c_{f}$ & $1.34 € / 1$ \\
$I_{f g}$ & $1.8 \mathrm{kgm}^{2}$ & $c_{e l}$ & $0.1 € / \mathrm{kWh}$ \\
$I_{\gamma=1: 12}$ & $1 \mathrm{kgm}^{2}$ & $s$ & $50000 \mathrm{~km} / \mathrm{year}$ \\
$\Delta t_{u p}$ & $2 \mathrm{~s}$ & $s o c_{\min }$ & 0.25 \\
$P_{\text {fric }}$ & $16 \mathrm{~kW}$ & $s o c_{\max }$ & 0.75 \\
\hline
\end{tabular}

which is gridded with 100 grid points to obtain the discrete set $\tilde{\mathcal{T}}_{I C E_{i \bar{\gamma}}}$. Efficiency is assigned to each grid value that gives the following set

$$
\begin{gathered}
\mathcal{N}_{I C E_{t \bar{\gamma}}}=\left\{\eta_{I C E}\left(\bar{\omega}_{I C E}(\bar{\gamma}), \bar{T}_{I C E}(\bar{\gamma})\right) \mid e_{o n}(t)\right. \\
\left.\wedge \bar{T}_{I C E}(\bar{\gamma}) \in \tilde{\mathcal{T}}_{I C E_{t \bar{\gamma}}}\right\} .
\end{gathered}
$$

4) The efficiencies for each of the $N$ time instances and each gear are arranged in a two-dimensional matrix $M \in \mathbb{R}^{N \times\left(\gamma_{\max }+1\right)}$ such that

$$
\begin{aligned}
& M(t, \bar{\gamma})= \begin{cases}\bar{\eta}(\bar{\gamma}), & \bar{\gamma} \in \mathcal{G}_{t} \wedge \text { cond }_{1} \\
0, & \bar{\gamma} \notin \mathcal{G}_{t}\end{cases} \\
& \operatorname{cond}_{1}=\left(e_{o n}(t) \wedge \bar{\eta}(\bar{\gamma})=\max \left\{\mathcal{N}_{\left.\left.I C E_{t \bar{\gamma}}\right\}\right)}\right.\right. \\
& \quad \vee\left(\neg e_{o n}(t) \wedge \bar{\eta}(\bar{\gamma})=\eta_{E M}\left(\bar{\omega}_{E M}(\bar{\gamma}), \bar{T}_{S}(\bar{\gamma}) / r_{E M}\right)\right)
\end{aligned}
$$

where zero efficiency has been assigned to infeasible gears. At a given time instance, feasible gear, and ICE off, the assigned matrix efficiency is the EM efficiency, while for the ICE turned on, the assigned matrix efficiency is the maximum efficiency of the grided torque split values in (A.2).

5) Gear is selected that maximizes ICE efficiency when the ICE is on, or otherwise it maximizes EM efficiency. During braking, gear is not changed, unless the previously selected feasible gear becomes infeasible at the current time instance. Mathematically, this can be expressed as

$$
\begin{aligned}
& \gamma(t)= \begin{cases}\bar{\gamma}, & \bar{\eta}(\bar{\gamma})=\max \{\tilde{M}(t, \bar{\gamma})\} \wedge \text { cond }_{2} \\
\gamma^{-}(t), & F_{\text {base }}(t)<0 \wedge \gamma^{-}(t) \in \mathcal{G}_{t}\end{cases} \\
& \operatorname{cond}_{2}=\left(F_{\text {base }}(t) \geq 0\right) \vee\left(F_{\text {base }}(t)<0 \wedge \gamma^{-}(t) \notin \mathcal{G}_{t}\right)
\end{aligned}
$$

where $\gamma^{-}(t)$ is the gear selected at the previous time instance. To prevent frequent gear shifts, instead of $M$, a filtered matrix $\tilde{M}$ is used, obtained by filtering the efficiency matrix with a two-dimensional moving average filter.
6) After gear has been selected at each time instance along the bus line, the convex problem is solved and the optimal energy buffer size is obtained. The powertrain is updated with the optimal buffer size, the demanded force $F_{\text {base }}(t)$ is computed and the procedure is repeated from 2) until the improvement in cost is smaller than some small positive number $\epsilon$.

Experiments showed that the gear selection procedure with $\epsilon=1 \times 10^{-3}$ usually ends in 2-3 iterations.

Note. The set of feasible gears $\mathcal{G}(t)$ in (A.1) must not be empty at any time instance, since otherwise the problem is infeasible.

\section{References}

[1] L. Guzzella, A. Sciarretta, Vehicle propulsion systems, introduction to modeling and optimization, 2nd Edition, Springer, Berlin, Heidelberg, 2007.

[2] On-line electric vehicle, Tech. rep., Korea Advanced Institute of Science and Technology (KAIST) (2009).

[3] AutoTram: Transport system of the future, Tech. rep., Fraunhofer Institute for Transportation and Infrastructure Systems IVI (2010).

[4] L. Johannesson, Predictive control of hybrid electric vehicles on prescribed routes, Ph.D. thesis, Chalmers University of Technology, Göteborg, Sweden (2009).

[5] T. C. Moore, HEV control strategy: Implications of performance criteria, system configuration and design, and component selection, in: Proceedings of the American Control Conference, Albuquerque, New Mexico, 1997.

[6] L. Triger, J. Paterson, P. Drozdz, Hybrid vehicle engine size optimization, in: SAE Future Transportation Technology Conference and Exposition, San Antonio, Texas, USA, 1993.

[7] M. R. Cuddy, K. B. Wipke, Analysis of the fuel economy benefit of drivetrain hybridization, in: SAE International Congress \& Exposition, Detroit, Michigan, 1997.

[8] V. Galdi, L. Ippolito, A. Piccolo, A. Vaccaro, A genetic-based methodology for hybrid electric vehicles sizing, Soft Computing - A Fusion of Foundations, Methodologies and Applications 5 (6) (2001) 451-457.

[9] X. Hu, Z. Wang, L. Liao, Multi-objective optimization of HEV fuel economy and emissions using evolutionary computation, in: SAE World Congress Detroit, Michigan, 2004.

[10] S. M. Lukic, A. Emadi, Effects of drivetrain hybridization on fuel economy and dynamic performance of parallel hybrid electric vehicles, IEEE Transactions on Vehicular Technology 53 (2) (2004) 385-389.

[11] J. M. Miller, P. J. McCleer, M. Everett, E. G. Strangas, Ultracapacitor plus battery energy storage system sizing methodology for HEV power split electronic CVT's, in: IEEE ISIE, Dubrovnik, Croatia, 2005.

[12] C. Holder, J. Gover, Optimizing the hybridization factor for a parallel hybrid electric small car, in: IEEE Vehicle Power and Propulsion Conference (VPPC), 2006.

[13] D. Rotenberg, A. Vahidi, I. Kolmanovsky, Ultracapacitor assisted powertrains: Modeling, control, sizing, and the impact on fuel economy, in: American Control Conference, Seattle, Washington, USA, 2008.

[14] R. Bellman, Dynamic Programming, Princeton Univ Pr, New Jersey, 1957.

[15] U. Zoelch, D. Schroeder, Dynamic optimization method for design and rating of the components of a hybrid vehicle, International Journal of Vehicle Design 19 (1) (1998) 1-13.

[16] M. Kim, H. Peng, Power management and design optimization of fuel cell/battery hybrid vehicles, Journal of Power Sources 165 (2) (2007) 819-832.

[17] O. Sundström, L. Guzzella, P. Soltic, Torque-assist hybrid electric powertrain sizing: From optimal control towards a sizing law, IEEE Transactions on Control Systems Technology 18 (4) (2010) 837-849.

[18] M. Kim, H. Peng, Combined control/plant optimization of fuel cell hybrid vehicles, in: Proceedings of the 2006 American Control Conference Minneapolis, Minnesota, USA, 2006.

[19] S. J. Moura, D. S. Callaway, H. K. Fathy, J. L. Stein, Tradeoffs between battery energy capacity and stochastic optimal power management in 
plug-in hybrid electric vehicles, Journal of Power Sources 195 (9) (2010) 2979-2988.

[20] N. Murgovski, J. Sjöberg, J. Fredriksson, A methodology and a tool for evaluating hybrid electric powertrain configurations, Int. J. Electric and Hybrid Vehicles 3 (3) (2011) 219-245.

[21] S. Boyd, L. Vandenberghe, Convex Optimization, Cambridge University Press, 2004.

[22] Y. Labit, D. Peaucelle, D. Henrion, SeDuMi interface 1.02: a tool for solving LMI problems with SeDuMi, IEEE International Symposium on Computer Aided Control System Design Proceedings (2002) 272-277.

[23] K. C. Toh, R. H. Tütüncü, M. J. Todd, On the implementation and usage of SDPT3 - a Matlab software package for semidefinite-quadratic-linear programming, version 4.0 (July 2006)

[24] Staff paper on the standardization of electric vehicle charging infrastructure, Tech. rep., California Air Resources Board (February 2001).

[25] A. Sciarretta, L. Guzzella, Control of hybrid electric vehicles, IEEE Control Systems Magazine 27 (2) (2007) 60-70.

[26] P. Pisu, G. Rizzoni, A comparative study of supervisory control strategies for hybrid electric vehicles, IEEE Transactions on Control Systems Technology 15 (3) (2007) 506-518.

[27] D. Ambühl, O. Sundström, A. Sciarretta, L. Guzzella, Explicit optimal control policy and its practical application for hybrid electric powertrains, Control Engineering Practice 18 (12) (2010) 1429-1439.

[28] M. Neuman, H. Sandberg, B. Wahlberg, A. Folkesson, Modelling and control of series HEVs including resistive losses and varying engine efficiency, in: SAE International, 2008

[29] I. Nowak, Relaxation and Decomposition Methods for Mixed Integer Nonlinear Programming, Birkhäuser Basel, 2005.

[30] M. Grant, S. Boyd, CVX: Matlab software for disciplined convex programming, version 1.21, http://cvxr.com/cvx (May 2010).

[31] J. Löfberg, YALMIP: A toolbox for modeling and optimization in Matlab, In Proceedings of the CACSD Conference, Taipei, Taiwan, http://users.isy.liu.se/johanl/yalmip (2004).

[32] S. J. Pachernegg, A closer look at the willans-line, SAE Technical Paper, 1969, doi:10.4271/690182.

[33] L. Guzzella, C. H. Onder, Introduction to Modeling and Control of Internal Combustion Engine Systems, Springer-Verlag, 2010.

[34] G. Rizzoni, L. Guzzella, B. M. Baumann, Unified modeling of hybrid electric vehicle drivetrains, IEEE/ASME Trans. Mechatron. 4 (3) (1999) $246-257$.

[35] X. Wei, G. Rizzoni, A scalable approach for energy converter modeling and supervisory control design, Proc. ASME Int. Mech. Eng. Congr. Expos. (2001) 1281-1288.

[36] S. Barsali, C. Miulli, A. Possenti, A control strategy to minimize fuel consumption of series hybrid electric vehicles, IEEE Transactions on Energy Conversion 19 (1) (2004) 187-195.

[37] J. Gao, F. Sun, H. He, G. G. Zhu, E. G. Strangas, A comparative study of supervisory control strategies for a series hybrid electric vehicle, in: IEEE Power and Energy Engineering Conference, 2009.

[38] X. Wei, L. Guzzella, V. I. Utkin, G. Rizzoni, Model-based fuel optimal control of hybrid electric vehicle using variable structure control systems, Journal of Dynamic Systems, Measurement, and Control 129 (1) (2007) 13-19.

[39] F. Mapelli, M. Mauri, D. Tarsitano, Energy control strategies comparison for a city car plug-in HEV, in: Industrial Electronics, IECON '09. 35th Annual Conference of IEEE., 2009.

[40] G. Lucente, M. Montanari, C. Rossi, Modelling of an automated manual transmission system, Mechatronics 17 (73-91).

[41] M. J. Nunney, Light and Heavy Vehicle Technology, ButterworthHeinemann, 2007.

[42] L. Gaines, R. Cuenca, Costs of lithium-ion batteries for vehicles, Tech. rep., Center for Transportation Research at Argonne National Labaratory, United States Department of Energy (May 2000).

[43] A. Wiederer, R. Philip, Policy options for electric vehicle charging infrastructure in c40 cities, Tech. rep. (2010).

[44] L. Johannesson, S. Pettersson, B. Egardt, Predictive energy management of a 4QT series-parallel hybrid electric bus, Control Engineering Practice 17 (12) (2009) 1440-1453.

[45] S. Boyd, N. Parikh, E. Chu, Distributed Optimization and Statistical Learning via the Alternating Direction Method of Multipliers, Now Publishers Inc, 2011.
[46] L. Johannesson, M. Asbogård, B. Egardt, Assessing the potential of predictive control for hybrid vehicle powertrains using stochastic Dynamic Programming, IEEE Transactions on Intelligent Transportation Systems 8 (1) (2007) 71-83.

[47] O. Sundström, L. Guzzella, A generic Dynamic Programming Matlab function, in: 18th IEEE International Conference on Control Applications, Saint Petersburg, Russia, 2009.

[48] O. Sundström, D. Ambühl, L. Guzzella, On implementation of Dynamic Programming for optimal control problems with final state constraints, Oil Gas Sci. Technol. 65 (1) (2009) 91-102. 